\title{
ON AN ANALOGUE OF TITCHMARSH'S DIVISOR PROBLEM FOR HOLOMORPHIC CUSP FORMS
}

\author{
NIGEL J. E. PITT
}

\section{INTRODUCTION}

A central question in analytic number theory is to understand the average value of arithmetical functions $\xi(n)$ at primes, which amounts to estimating the sum

$$
\sum_{n \leq X} \Lambda(n) \xi(n)
$$

where $\Lambda$ denotes the von Mangoldt function, given by $\Lambda(n)=\log p$ if $n=p^{\alpha}$ for some prime $p$, and $\Lambda(n)=0$ if not. Since

$$
\sum_{n=1}^{\infty} \Lambda(n) n^{-s}=-\frac{\zeta^{\prime}(s)}{\zeta(s)}
$$

the estimation of (1.1) for $\xi=1$ is related to the location of the zeros of $\zeta$, which is the classical approach to the prime number theorem, and for multiplicative $\xi$ in general the problem can be related to the properties of a corresponding $L$-function in a similar way. (Chapter 5 of [13] contains a comprehensive discussion of this and related issues).

A shift in the argument of $\xi$ destroys the multiplicativity, however, and this approach to estimating (1.1) is not applicable. A classical example is Titchmarsh's divisor problem, which considers the average value of the divisor function $\tau(n)$ at shifted primes, in other words, the estimation of

$$
\mathscr{T}(X)=\sum_{n \leq X} \Lambda(n) \tau(n-l)
$$

where $l>0$ is fixed. In this case the importance of the shift becomes even clearer when one notes that $\tau(p)=2$, so the problem for $l=0$ is the same as the case $\xi=1$ in (1.1).

Similar considerations apply to sums

$$
\sum_{n \leq X} \mu(n) \xi(n)
$$

Received by the editors August 8, 2011 and, in revised form, November 18, 2011, and April $30,2012$.

2010 Mathematics Subject Classification. Primary 11F11, 11F30; Secondary 11F72, 11N37.

Key words and phrases. Titchmarsh divisor problem, Möbius randomness, Kloosterman sums, Kuznetsov formula, shifted convolution. 
where $\mu(n)$ denotes the Möbius function, given by $\mu(1)=1, \mu(n)=(-1)^{k}$ if $n$ is the product of $k$ distinct primes, and $\mu(n)=0$ otherwise. Since

$$
\sum_{n=1}^{\infty} \mu(n) n^{-s}=\frac{1}{\zeta(s)}
$$

the estimation of (1.4) for $\xi=1$ is again related to the location of the zeros of $\zeta$; the prime number theorem is equivalent to (1.4) being $o(X)$ and the Riemann hypothesis is equivalent to it being $O\left(X^{1 / 2+\varepsilon}\right)$, and as in the case of (1.1) its estimation for multiplicative $\xi$ is related to the theory of the corresponding $L$-function. More generally, however, it is widely believed that the changes of sign in $\mu(n)$ should be independent of those in any "reasonable" function $\xi$, multiplicative or not, which would cause cancellation between terms and make (1.4) small compared to $\sum|\xi(n)|$ as $X \rightarrow \infty$; for instance, if $|\xi(n)|=1$ for all $n$, then (1.4) should be $o(X)$. This is often called the Möbius randomness principle, a precise statement requiring a definition of "reasonable". (Sarnak discusses this in 24], suggesting that (1.4) should be $o(X)$ whenever $\xi$ arises from a dynamical system of zero entropy).

We will consider these problems for $\xi(n)=a(n-1)$, where

$$
f(z)=\sum_{n=1}^{\infty} A(n) e(n z)=\sum_{n=1}^{\infty} a(n) n^{(k-1) / 2} e(n z)
$$

is a holomorphic cusp form of weight $k$ for the modular group $\mathrm{PSL}_{2}(\mathbb{Z})$. Here, as throughout the following, $e(\xi)=e^{2 \pi i \xi}$. The coefficients $A(n)$ are bounded by $O\left(n^{(k-1) / 2} \tau(n)\right)$, due to Deligne [5], so the normalised coefficients $a(n)$ are bounded by $O(\tau(n))$, and the sums

$$
\mathscr{T}_{f}(X):=\sum_{n \leq X} \Lambda(n) a(n-1), \mathscr{M}_{f}(X):=\sum_{n \leq X} \mu(n) a(n-1)
$$

are both trivially bounded by $O\left(X^{1+\varepsilon}\right)$. Were we to suppose $f$ to be a Hecke eigenform, which is not necessary in what follows, then these would be cases of the two problems discussed above for a multiplicative function with a shift. The main result of this paper is the following upper bound.

Theorem 1.1. If $X>1$, then $\mathscr{T}_{f}(X) \ll X^{391 / 392+\varepsilon}$ for any $\varepsilon>0$, where the implied constant depends on $f$ and $\varepsilon$. Furthermore, the same bound holds with $\mathscr{M}_{f}(X)$ in place of $\mathscr{T}_{f}(X)$.

The bound for $\mathscr{M}_{f}(X)$ can be viewed as a case of Möbius randomness, since it is better than trivial by a power of $X$ and hence shows substantial cancellation between terms. The bound for $\mathscr{T}_{f}(X)$ should be compared with work on the sum (1.3), since $\tau(n)$ can be viewed as the Fourier coefficients of an automorphic function, so $\mathscr{T}(X)$ and $\mathscr{T}_{f}(X)$ are analogous. Titchmarsh's original work in 28 and [29] shows that $\mathscr{T}(X)$ is asymptotic to $c_{1}(l) X \log X$ where $c_{1}(l)$ is a constant depending on $l$, conditionally on the generalised Riemann hypothesis; this result was later proved unconditionally by Linnik [20, and also considered in [11] and [23. The best known result at present is

$$
\mathscr{T}(X)=c_{1}(l) X \log X+c_{2}(l) X+O\left(X(\log X)^{-A}\right)
$$

for any $A>0$, where the implied constant depends only on $A$ and $l$, due to Bombieri, Friedlander and Iwaniec [4, and to Fouvry [8], independently. In the case of $\mathscr{T}_{f}(X)$ there is no main term since the coefficients are oscillatory, so upper bounds for $\mathscr{T}_{f}$ 
should be compared with the error terms in asymptotic bounds for (1.3). As such, Theorem 1.1 is better than trivial by a power of $X$, which is significantly stronger than the arbitrary power of $\log X$ in (1.6).

The proof of Theorem 1.1 uses well-known combinatorial ideas to decompose the sums (1.5) into expressions of the general form

$$
\sum_{n_{1}, \ldots, n_{k}} G\left(n_{1} \ldots n_{k}\right) a\left(n_{1} \ldots n_{k}-1\right)
$$

for small values of $k$ and appropriate smoothing functions $G$, together with sums which can be considered as bilinear forms. If any of the variables $n_{j}$ is sufficiently long then the well-known Voronoi-type summation formula for the Fourier coefficients can be used to estimate the sum adequately; in practice this already covers $k=1$ and $k=2$. On the other hand, the ranges for which available bounds for bilinear forms are non-trivial determines how many values of $k$ must be treated separately; in our case this includes $k=3$, for which results are available from [22], including the bound 1

$$
\sum_{n \leq X} \tau_{3}(n) a(r n-1) \ll r^{5 / 24} X^{71 / 72+\varepsilon},
$$

where the implied constant depends on $f$ and $\varepsilon$. In fact, rather than apply (1.8) directly we will use a related bound from 22. The bound (1.8) and the related estimates in 22 are proved using methods quite different from those discussed here, an essential step being the use of the Riemann hypothesis for varieties in the estimation of a complete exponential sum over a finite field in three variables.

The bilinear forms that appear can be estimated in slightly different ways depending on the properties of the coefficients; although we need only one of them, for completeness we collect these bounds in the following theorem.

Theorem 1.2. Let $M, N>1$ and let $\alpha_{m}, \beta_{n}$ denote arbitrary complex coefficients. Then for any $\varepsilon>0$ the bilinear form

$$
\mathscr{B}(\alpha, \beta)=\sum_{m \leq M} \sum_{n \leq N} \alpha_{m} \beta_{n} A(m n-1)
$$

can be bounded by

$$
\mathscr{B}(\alpha, \beta) \ll_{f, \varepsilon}\|\alpha\|\|\beta\|\left\{M^{3 / 8} N^{1 / 2}+M^{3 / 4} N^{3 / 8}\right\}(M N)^{(k-1) / 2+\varepsilon}
$$

where the implied constant depends on the form $f$ and on $\varepsilon$, and where

$$
\|\alpha\|^{2}=\sum_{m \leq M}\left|\alpha_{m}\right|^{2},\|\beta\|^{2}=\sum_{n \leq N}\left|\beta_{n}\right|^{2} .
$$

If the coefficients $\alpha_{m}$ are supported on squarefree values of $m$, then (1.9) can be improved to

$$
\mathscr{B}(\alpha, \beta) \ll_{f, \varepsilon}\|\alpha\|\|\beta\|\left\{N^{1 / 2}+M^{3 / 4} N^{3 / 8}\right\}(M N)^{(k-1) / 2+\varepsilon} .
$$

\footnotetext{
${ }^{1}$ We take the opportunity to correct an error on page 403 of [22], where $h(t)$ is supported on $t \sim r x$ and not $t \sim x$ as stated. This affects the calculations on page 404, and the exponent in $r$ in Theorem 2 of [22], which should be corrected as in (1.8) here. The exponent in $X$ is unchanged, whereas the exponent is weaker in $r$; note, however, that the corrected bound is valid in a wider range than as originally stated. Theorem 1 and the other results proved prior to p. 403 in 22 ] remain unchanged.
} 
If $\alpha_{m} \ll m^{\varepsilon}$ for any $\varepsilon>0$, then (1.9) can be improved to

$$
\mathscr{B}(\alpha, \beta) \ll_{f, \varepsilon}\|\beta\|\left\{M^{3 / 4} N^{1 / 2}+M^{5 / 4} N^{3 / 8}\right\}(M N)^{(k-1) / 2+\varepsilon} .
$$

Note that Theorem 1.2 has been stated in terms of the unnormalised coefficients $A(n)$, for convenience. We will require the bound (1.11) in terms of the normalised coefficients $a(n)$, and it is convenient to state the following corollary separately.

Corollary 1.3. Let $M_{1}, M_{2}, X, Y>1$ with $M_{1}<M_{2}<X$ and $Y<X$ and let $\alpha_{m}, \beta_{n}$ denote complex coefficients bounded by $O\left(m^{\varepsilon}\right)$ and $O\left(n^{\varepsilon}\right)$, respectively, for any $\varepsilon>0$. Let $g(x)$ be a $\mathscr{C}^{\infty}$ function on $(-\infty, \infty)$, identically one on $[X, 2 X]$ and supported on $[X-Y, 2 X+Y]$, with derivatives bounded by $g^{(\nu)}(x) \ll Y^{-\nu}$ for $\nu=0,1,2 \ldots$ Then for any $\varepsilon>0$,

$$
\mathscr{B}_{g}(\alpha, \beta)=\sum_{M_{1}<m \leq M_{2}} \sum_{n} \alpha_{m} \beta_{n} g(m n) a(m n-1)
$$

can be bounded by

$$
\mathscr{B}_{g}(\alpha, \beta) \ll_{f, \varepsilon}\left\{X M_{1}^{-1 / 4}+X^{7 / 8} M_{2}^{3 / 8}\right\} X^{\varepsilon}
$$

where the implied constant depends on the form $f$ and on $\varepsilon$.

It is now easy to see why the case $k=3$ of (1.7) must be handled separately using (1.8) or something similar. To obtain a saving of a power of $X$ using (1.13) we require $M_{1}$ to be at least $X^{\delta_{1}}$ and $M_{2}$ at most $X^{1 / 3-\delta_{2}}$ for some $\delta_{1}, \delta_{2}>0$. In factorisations $n=n_{1} n_{2} n_{3}$ for $n \asymp X$ the worst case will be when all factors are about the same size, approximately $X^{1 / 3}$, for which Corollary 1.3 gives a trivial bound. For the sum (1.7) with $k \geq 4$, however, some factor or product of factors will be of an appropriate size unless one or more factors are extremely small, in which case $k$ is, in effect, reduced. (These small factors can be viewed as $r$ in (1.8), for instance.)

In fact, the particular combinatorial method used is a partially smoothed version of Vaughan's identity from 30, which is iterated three times. This does not give sums precisely of the form (1.7), although they are close enough for the loose description above to make sense. Perhaps other more direct combinatorial approaches might be used instead; however, the author was unable to apply Linnik's identity ([20], see also [13]) directly due to difficulties with large values of $k$, which made necessary the slightly cumbersome approach used below.

The proof of Theorem 1.2 begins with an application of Cauchy's inequality to remove one set of arbitrary coefficients, say $\beta_{n}$, and the resulting sum over $n$ is then treated as a shifted convolution of the Fourier coefficients and bounded using the following theorem.

Theorem 1.4. Let

$$
f(z)=\sum_{n=1}^{\infty} A(n) e(n z), g(z)=\sum_{n=1}^{\infty} B(n) e(n z)
$$


be two cusp forms of weight $k$ for $\mathrm{PSL}_{2}(\mathbb{Z})$ and let $m_{1}, m_{2}$ be distinct squarefree integers. Defining $d=\left(m_{1}, m_{2}\right)$ and $D=\left(m_{1}-m_{2}, d^{\infty}\right)$ we have the bound

$$
\begin{aligned}
& \sum_{n=1}^{\infty} e^{-4 \pi n / N} A\left(m_{1} n-1\right) \overline{B\left(m_{2} n-1\right)} \\
& \ll\left\{D d^{-1 / 2}\left(m_{1} m_{2} N\right)^{1 / 2}\right. \\
& \left.\quad+D^{1 / 4}\left(m_{1} m_{2}\right)^{1 / 4} N^{3 / 4}\right\}\left(m_{1} m_{2} N^{2}\right)^{(k-1) / 2+\varepsilon}
\end{aligned}
$$

for any $\varepsilon>0$, where the implied constant depends on the forms and on $\varepsilon$.

Shifted convolutions have long been studied, starting with the case $m_{1}=m_{2}=1$ considered by Selberg [27] who linked the meromorphic properties of an associated Dirichlet series to the spectral theory of the Laplacian; Good [9] later considered its growth. These ideas, and their analogues for Maass forms, have been used and extended by various authors, including improvements using modern results on spectral theory such as Kim and Sarnak's work towards the Ramanujan and Selberg conjectures [18]. We refer the reader to [1] and [25] for detailed discussions of these issues, also citing the papers [2, 17, [10], [12], [16], 17], 26] as some of the other contributions to this area. We will apply Theorem 1.4 in the proofs of the other theorems, but observe that the same ideas can be used to bound more general shifted convolutions. Since these may be of independent interest we state them in the form of another theorem.

Theorem 1.5. Let $f(z)$ and $g(z)$ be cusp forms of weight $k$ for $\operatorname{PSL}_{2}(\mathbb{Z})$ as above with normalised Fourier coefficients $a(n)$ and $b(n)$, and let $G\left(x_{1}, x_{2}\right)$ be compactly supported on $\left[X_{1}, 2 X_{1}\right] \times\left[X_{2}, 2 X_{2}\right]$ with derivatives bounded by

$$
G^{\left(\nu_{1}, \nu_{2}\right)}\left(x_{1}, x_{2}\right) \ll\left(Z_{1} / X_{1}\right)^{\nu_{1}}\left(Z_{2} / X_{2}\right)^{\nu_{2}}
$$

for some $Z_{1}, Z_{2} \geq 1$ and all $\nu_{1}, \nu_{2} \geq 0$. Further, let $m_{1}, m_{2}, h$ be positive integers with $\left(m_{1}, m_{2}\right)=1$ and $h<m_{1} m_{2}$, and define the shifted convolution

$$
\mathscr{D}_{G}\left(m_{1}, m_{2}, h\right):=\sum_{m_{1} k_{1}-m_{2} k_{2}=h} G\left(k_{1}, k_{2}\right) a\left(k_{1}\right) \overline{b\left(k_{2}\right)} .
$$

Then

$$
\begin{aligned}
& \mathscr{D}_{G}\left(m_{1}, m_{2}, h\right) \ll\left(\frac{m_{1} X_{1}}{Z_{1}^{2}}+\frac{m_{2} X_{2}}{Z_{2}^{2}}\right)^{1 / 2} Z^{1 / 4}\left(Z_{1} Z_{2}\right)^{1 / 2} \\
&+\left.\left(\frac{m_{1} X_{1}}{Z_{1}^{2}}+\frac{m_{2} X_{2}}{Z_{2}^{2}}\right)^{1 / 4}\left(\frac{X_{1} X_{2}\left(m_{1} m_{2}, h\right)}{m_{1} m_{2}}\right)^{1 / 4}\right\} \\
& \times Z^{3 / 2}\left(Z_{1} Z_{2}\right)^{5 / 2}\left(X_{1} X_{2}\right)^{\varepsilon}
\end{aligned}
$$

for any $\varepsilon>0$, where $Z=\max \left\{Z_{1}, Z_{2}\right\}$.

Theorems 1.4 and 1.5 are really two versions of the same bound, as can be seen in the case $\left(m_{1}, m_{2}\right)=1$, for which the sum in Theorem 1.4 can be written as

$$
e^{-2 \pi\left(1 / m_{1} N+1 / m_{2} N\right)} \sum_{\substack{k_{1}, k_{2}=1 \\ m_{1} k_{2}-m_{2} k_{1}=m_{2}-m_{1}}}^{\infty} e^{-2 \pi k_{1} / m_{1} N-2 \pi k_{2} / m_{2} N} A\left(k_{1}\right) \overline{B\left(k_{2}\right)} .
$$


Renormalising, this is clearly a case of Theorem 1.5. To compare with other known results, we consider the special case where $Z_{1}=Z_{2}=1$, for which Theorem 1.5 reduces to

$$
\begin{aligned}
\mathscr{D}_{G}\left(m_{1}, m_{2}, h\right) \ll\{ & \left(m_{1} X_{1}+m_{2} X_{2}\right)^{1 / 2} \\
& \left.+\left(m_{1} X_{1}+m_{2} X_{2}\right)^{1 / 4}\left(\frac{X_{1} X_{2}}{m_{1} m_{2}}\right)^{1 / 4}\left(m_{1} m_{2}, h\right)^{1 / 4}\right\}\left(X_{1} X_{2}\right)^{\varepsilon}
\end{aligned}
$$

and for which Blomer [1] has shown the estimate

$$
\mathscr{D}_{G}\left(m_{1}, m_{2}, h\right) \ll\left(m_{1} X_{1}+m_{2} X_{2}\right)^{1 / 2+\theta+\varepsilon}
$$

for any $\theta$ such that the Hecke eigenvalues $\lambda(n)$ are bounded by $|\lambda(n)| \leq n^{\theta} \tau(n)$. Conjecturally the bound holds with $\vartheta=\varepsilon$; the best bound known at present has $\theta=7 / 64$, due to Kim and Sarnak [18. Note that we have stated Blomer's result in less generality than he actually shows for easier comparison with Theorem 1.5. (See [1] for details). Note that (1.16) is completely uniform in $h$ as large as $m_{1} X_{1}+m_{2} X_{2}$, whereas we are only interested in small values of $h$, and in fact, make use of this small size in the proofs of Theorems 1.4 and 1.5. The essential point, however, is that these bounds improve on (1.16) only when $m_{1}$ and $m_{2}$ are large. For instance, in the most classical case where $m_{1}=m_{2}=1$ and $X_{1}=X_{2}=X$, Theorem 1.5 gives $\mathscr{D}_{G} \ll X^{3 / 4+\varepsilon}$, which is much weaker than the bound $\mathscr{D}_{G} \ll X^{1 / 2+\theta+\varepsilon}$ implied by (1.16). On the other hand, if $\left(m_{1} m_{2}, h\right)=1$ and $X_{1}=X_{2}=X$, then Theorem 1.5 will be better than $(1.16)$ whenever $\left(m_{1}+m_{2}\right)^{1 / 4}\left(m_{1} m_{2}\right)^{-1 / 4} X^{3 / 4+\varepsilon}$ is smaller than $\left(m_{1}+m_{2}\right)^{1 / 2+\theta+\varepsilon} X^{1 / 2+\theta+\varepsilon}$, which is the case in the range of uniformity in $m_{1}$ and $m_{2}$ that we need for our application to Theorem 1.1, as discussed above. Note, however, that if the Selberg eigenvalue conjecture were known, then the second term in Theorem 1.4 or 1.5 can be dropped, and if the generalised Ramanujan conjecture $|\lambda(n)| \ll n^{\varepsilon}$ were also known, then all the bounds would agree.

The restriction to coprime $m_{1}$ and $m_{2}$ in the previous paragraph is evidently not realistic for the application to Theorem 1.2. In fact, bounds similar to Theorem 1.5 for the coprime case were already proved in [21], but the methods used there do not extend to the general case, and were therefore inadequate to study the bilinear forms in Theorem 1.2. Issues arising in the cases where $\left(m_{1}, m_{2}\right)>1$ affect several steps in the proofs below, including the discussion of a particular Kloosterman sum in Section 3. Also, the proof uses Jutila's variant of the circle method (see [14] and 15]) which allows more flexibility in its application than does the more traditional decomposition of the unit interval using Farey fractions, for instance, since the denominators of the fractions used can be chosen conveniently. This helps to overcome these issues of non-coprimality. Following this the modular relations for the cusp forms are applied to produce sums of Kloosterman sums, for which we prove the following bound.

Theorem 1.6. Let $n, r$, and $s$ be positive integers with $0<n<r s$ and $(r, s)=1$, and let $F\left(x_{1}, x_{2}\right)$ be a real-valued $\mathscr{C}^{\infty}$ function compactly supported on $M<x_{1} \leq$ $2 M$ and $C<x_{2} \leq 2 C$, with derivatives of all orders bounded by

$$
F^{\left(\nu_{1}, \nu_{2}\right)}\left(x_{1}, x_{2}\right) \ll Z^{\nu_{1}+\nu_{2}} M^{-\nu_{1}} C^{-\nu_{2}}
$$


for some $Z \geq 1$ and all $\nu_{1}, \nu_{2} \geq 0$. There exists an absolute constant $X_{0} \in(0,1)$ such that if $M n / r s^{2} C^{2}, n / s C^{2}<X_{0}$, then for any $\varepsilon>0$ and any complex coefficients $\alpha_{n}$,

$$
\begin{aligned}
\sum_{(c, r)=1} \frac{1}{c} \sum_{m} \alpha_{m} F(m, c) S(m \bar{r}, \pm n ; s c) & \\
\ll\|\alpha\|\{ & s \sqrt{r}\left(Z^{3 / 4}+\sqrt{M / r s}\right) Z^{7 / 4} \\
& \left.+\left(s^{3 / 4}+M^{1 / 4} s^{1 / 2} r^{-1 / 4}\right)(n, r s)^{1 / 4} C^{1 / 2} Z^{3 / 2}\right\}(r s C Z)^{\varepsilon}
\end{aligned}
$$

where the implied constant depends on $\varepsilon$ alone.

The proof of Theorem 1.6 uses Kuznetsov's formulas [19, which express sums of Kloosterman sums in terms of spectral data. The formulas for Hecke congruence groups, together with some necessary estimates for Fourier coefficients of automorphic forms, can be found in [6] by Deshouillers and Iwaniec. We recall the main results, referring to $[6$ for all definitions not given here. Let $\mathfrak{a}$ and $\mathfrak{b}$ be two cusps for the Hecke congruence group $\Gamma=\Gamma_{0}(q)$ and let $\sigma_{\mathfrak{a}}$ and $\sigma_{\mathfrak{b}}$ be the corresponding scaling matrices. For any integers $m, n$ and any $\gamma$ real and positive such that there

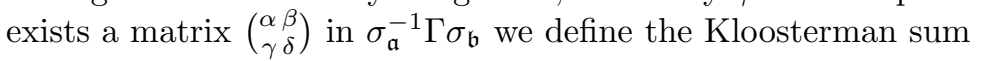

$$
S_{\mathfrak{a} \mathfrak{b}}(m, n ; \gamma)=\sum_{\delta(\bmod \gamma \mathbb{Z})}^{*} e\left(m \frac{\alpha}{\gamma}+n \frac{\delta}{\gamma}\right)
$$

where the sum is over all $\delta$ modulo $\gamma \mathbb{Z}$ for which there exists such a matrix, and following the notation in [6] we write the Fourier expansions of normalised holomorphic cusp forms of weight $k$, Maass cusp forms and Eisenstein series in the forms

$$
\begin{gathered}
j_{\sigma_{\mathfrak{a}}}^{-k}(z) f_{j k}\left(\sigma_{\mathfrak{a}} z\right)=\sum_{n \geq 1} \psi_{j k}(\mathfrak{a}, n) e(n z), \\
u_{j}\left(\sigma_{\mathfrak{a}} z\right)=y^{1 / 2} \sum_{n \neq 0} \varrho_{j \mathfrak{a}}(n) K_{i t_{j}}(2 \pi|n| y) e(n x),
\end{gathered}
$$

and

$$
\begin{aligned}
E_{\mathfrak{a}}\left(\sigma_{\mathfrak{b}} z, s\right)=\delta_{\mathfrak{a} \mathfrak{b}} y^{s}+ & \frac{\pi^{1 / 2} \Gamma(s-1 / 2)}{\Gamma(s)} \varphi_{\mathfrak{a} \mathfrak{b} 0}(s) y^{1-s} \\
& +\frac{2 \pi^{s} y^{1 / 2}}{\Gamma(s)} \sum_{n \neq 0} \varphi_{\mathfrak{a} \mathfrak{b} n}(s)|n|^{s-1 / 2} K_{s-1 / 2}(2 \pi|n| y) e(n x) .
\end{aligned}
$$

As in [6], note that, without loss of generality, we may suppose that $u_{j}$ is real, and hence $\varrho_{j \mathfrak{a}}(-m)=\overline{\varrho_{j \mathfrak{a}}(m)}$, and that $\varrho_{\mathfrak{a} \mathfrak{b}(-m)}(1 / 2+i t)=|m|^{-2 i t} \overline{\varrho_{\mathfrak{a} \mathfrak{b} m}(1 / 2+i t)}$ since the Eisenstein series is real for real $s$. For any $\mathscr{C}^{\infty}$ function $f$ of compact support 
in $(0, \infty)$ and any $m, n>0$, the Kuznetsov formula states

$$
\begin{aligned}
\sum_{\gamma} \frac{1}{\gamma} S_{\mathfrak{a} \mathfrak{b}}(m, n ; \gamma) f\left(\frac{4 \pi \sqrt{m n}}{\gamma}\right) \\
=\frac{1}{\pi} \sum_{\substack{k \equiv 0 \\
(\bmod 2)}} \sum_{j} \frac{i^{k}(k-1) !}{(4 \pi)^{k-1}} \bar{\psi}_{j k}(\mathfrak{a}, m) \psi_{j k}(\mathfrak{b}, n) \tilde{f}(k-1) \\
\quad+\sum_{j \geq 1} \frac{\bar{\varrho}_{j \mathfrak{a}}(m) \varrho_{j \mathfrak{b}}(n)}{\cosh \pi t_{j}} \hat{f}\left(t_{j}\right) \\
\quad+\frac{1}{\pi} \sum_{\mathfrak{c}} \int_{-\infty}^{\infty}\left(\frac{m}{n}\right)^{-i t} \bar{\varphi}_{\mathfrak{c a} m}\left(\frac{1}{2}+i t\right) \varphi_{\mathfrak{c} \mathfrak{b} n}\left(\frac{1}{2}+i t\right) \hat{f}(t) d t
\end{aligned}
$$

Here $\mathfrak{c}$ ranges over a complete set of inequivalent cusps, and the integral transforms are given by

$$
\tilde{f}(l)=\int_{0}^{\infty} J_{l}(y) f(y) \frac{d y}{y}, \hat{f}(t)=\frac{\pi}{\sinh \pi t} \int_{0}^{\infty} \frac{J_{2 i t}(x)-J_{-2 i t}(x)}{2 i} f(x) \frac{d x}{x} .
$$

A second form of the identity is valid when $m$ and $n$ have differing signs; specifically, for $m, n>0$

$$
\begin{aligned}
\sum_{\gamma} \frac{1}{\gamma} S_{\mathfrak{a} \mathfrak{b}}(m,- & n ; \gamma) f\left(\frac{4 \pi \sqrt{m n}}{\gamma}\right)=\sum_{j \geq 1} \frac{\varrho_{j \mathfrak{a}}(m) \varrho_{j \mathfrak{b}}(n)}{\cosh \pi t_{j}} \check{f}\left(t_{j}\right) \\
& +\frac{1}{\pi} \sum_{\mathfrak{c}} \int_{-\infty}^{\infty}(m n)^{i t} \varphi_{\mathfrak{c} \mathfrak{a} m}\left(\frac{1}{2}-i t\right) \varphi_{\mathfrak{c} \mathfrak{b} n}\left(\frac{1}{2}+i t\right) \check{f}(t) d t
\end{aligned}
$$

where

$$
\check{f}(t)=\frac{2}{\pi} \cosh \pi t \int_{0}^{\infty} K_{2 i t}(x) f(x) \frac{d x}{x} .
$$

We refer the reader to [6] for further details and the proofs of (1.17) and (1.18). We will use these formulas in two separate situations, in each case $f(x)$ will be supported on $x \asymp X$ for some $X>0$, with

$$
f^{(\nu)}(x) \ll(Z / X)^{\nu}
$$

for some $Z \geq 1$ and $\nu$ from 0 to at least 2 . The required estimates for the corresponding transforms $\hat{f}(t), \check{f}(t)$ and $\tilde{f}(t)$ are established in Lemma 2.1.

The sum formula in itself is of little use without adequate bounds for the terms that appear. Again citing [6], for $T \geq 1, N \geq 1 / 2$ and $\varepsilon>0$, each of the three quantities

$$
\sum_{\substack{2 \leq k \leq T \\ \text { even }}} \frac{(k-1) !}{(4 \pi)^{k-1}} \sum_{j}\left|\sum_{n \sim N} \alpha_{n} \bar{\psi}_{j k}(\mathfrak{a}, n)\right|^{2}, \sum_{\left|t_{j}\right| \leq T} \frac{1}{\cosh \pi t_{j}}\left|\sum_{n \sim N} \alpha_{n} \varrho_{j \mathfrak{a}}(n)\right|^{2},
$$

$$
\sum_{\mathfrak{c}} \int_{-T}^{T}\left|\sum_{n \sim N} \alpha_{n} n^{i t} \varphi_{\mathfrak{c a} n}\left(\frac{1}{2}+i t\right)\right|^{2} d t
$$


is bounded above by $O\left(\|\alpha\|^{2}\left(T^{2}+\mu(\mathfrak{a}) N^{1+\varepsilon}\right)\right)$ where $\mu(\mathfrak{a})=(w, q / w) q^{-1}$ for $\mathfrak{a}=$ $u / w$ and the implied constant depends on $\varepsilon$ alone. These estimates are known as the large sieve inequalities. For the exceptional eigenvalues stronger results are known; again from [6], for any $X \geq 1$ and $\varepsilon>0$,

$$
\sum_{\lambda_{j}-\text { exc }} X^{2 i t_{j}}\left|\sum_{n \sim N} \alpha_{n} \varrho_{j \mathfrak{a}}(n)\right|^{2} \ll\|\alpha\|^{2}(1+\sqrt{\mu(\mathfrak{a}) N X})\left(1+\sqrt{\mu(\mathfrak{a}) N^{1+\varepsilon}}\right)
$$

where $i t_{j}>0$ and the implied constant depends on $\varepsilon$ alone. Note that, as remarked in [6] where this bound is proved, this is better than the large sieve inequalities when $\mu(\mathfrak{a}) N<1$.

The proof of Theorem 1.6 is broken into two parts; the treatment of the regular spectrum, that is, the eigenfunctions with eigenvalue $\lambda_{j} \geq 1 / 4$ together with the continuous spectrum and the holomorphic forms, and the exceptional spectrum, corresponding to eigenvalues $\lambda_{j}<1 / 4$. The treatment of the regular spectrum is standard, being essentially the same as results from [6], and is an application of the formulas (1.17) and (1.18) and the large sieve inequalitites (1.20). It differs from the treatment of an analogous sum in [1] in using the large sieve inequalities in place of Kim and Sarnak's bound $\theta \leq 7 / 64$ (see [18]) which is beneficial since $n$ is small compared to the level of the group appearing. The treatment of exceptional eigenvalues is also different, using Weil's bound for the Kloosterman sums. This is not a direct application such as used by Duke, Friedlander and Iwaniec in 7] (since the resulting bound in Theorem 1.4 would be weaker by a factor of $\left.\left(m_{1} m_{2}\right)^{1 / 2}\right)$ but uses Cauchy's inequality followed by a second application of Kuznetsov's formulas, which produces Kloosterman sums of moduli in a different range, and which is efficient enough to prove Theorem 1.6; the argument is closely related to ideas from [6].

A summary of the structure of the proof, and of the paper, is as follows. In Section 9 three iterations of a smoothed form of Vaughan's identity (Lemma 9.1) are applied to the sums $\mathscr{T}_{f}(X)$ and $\mathscr{M}_{f}(X)$ to decompose them into smoothed sums of the form (1.7) for $k=1,2,3$, together with bilinear forms of the form $\mathscr{B}_{g}(\alpha, \beta)$ from Corollary 1.3. The sums (1.7) can be estimated for $k=1$ or $k=2$ using a Voronoi-type summation formula (discussed in Section 8) and the case $k=3$ is treated using bounds from [22, as discussed above. The required bounds from Corollary 1.3 are shown in Sections 6 and 7, and follow from the estimate for the shifted convolution in Theorem 1.4, itself proved in Section 5. This depends not only on the bound in Theorem 1.6 for sums of Kloosterman sums, proved in Section 2 , but also on the properties of some specific Kloosterman sums from Section 3, and a smoothed version of Jutila's version of the circle method established in Section 4. Finally, some bounds for integral transforms are stated in Lemma 2.1, and proved in Section 10.

\section{Sums of Kloosterman sums and the proof of Theorem 1.6}

The integral transforms appearing in (1.17) and (1.18) are bounded in various ranges in the following lemma.

Lemma 2.1. Let $X>0, Z \geq 1, k \in \mathbb{N}$. If $f$ is supported on $[X, 2 X]$ with derivatives of orders $\nu=0,1,2, \ldots, 2 k$ bounded by $f^{(\nu)}(x) \ll(Z / X)^{\nu}$, then the 
following bounds hold. For any real $t$ and any $l \leq k$,

$$
\hat{f}(t), \check{f}(t), \tilde{f}(t) \ll\left(\frac{Z^{2}+t^{2}}{X^{2}}\right)^{l}\left(\frac{1+|\log (X / Z)|}{1+X / Z}\right) .
$$

For all real $t$ with $|t|>1$,

$$
\hat{f}(t), \check{f}(t), \tilde{f}(t) \ll \frac{Z}{|t|}\left(\frac{1}{|t|^{1 / 2}}+\frac{X}{|t|}(1+\log |t|)\right),
$$

and for all real $t$ such that $|t|>\max \{2 X, 1\}$ and for all $j \leq 2 k$,

$$
\hat{f}(t), \check{f}(t), \tilde{f}(t) \ll\left(\frac{Z}{|t|}\right)^{j}\left(\frac{1}{|t|^{1 / 2}}+\frac{X}{|t|}(1+\log |t|)\right) .
$$

For exceptional eigenvalues $\lambda=1 / 4+(i t)^{2}$ we take $t \in(0,1 / 2)$ and

$$
\hat{f}(i t), \check{f}(i t) \ll \begin{cases}\frac{Z}{X} & \text { if } Z<X, \\ \left\{1+\log \frac{Z}{X}\right\}\left(\frac{Z}{X}\right)^{2 t} & \text { if } X<Z .\end{cases}
$$

Furthermore, if $t \in(0,1 / 4), f$ is non-negative and $\int f(x) d x / x \gg 1$, then there exists an absolute constant $X_{0} \in(0,1)$ such that

$$
\hat{f}(i t), \check{f}(i t) \gg X^{-2 t}
$$

if $X<X_{0}$. The implied constants in (2.1) and (2.3) depend only on $k$, whereas those in (2.2) and (2.4) are absolute.

Similar bounds have been given elsewhere in the literature (Lemma 7.1 in [6, see also Lemma 2.4 in [1] and Lemma 2.1 in 3]). They are stated here in more generality than we need; in particular, we only use the case $X<1$, and only consider congruence groups. These have exceptional eigenvalues bounded away from zero and hence $t$ bounded away from $1 / 2$ in (2.4). Nonetheless, we include the general case as of independent interest.

Some differences from the bounds in the literature should be noted. The logarithm in (2.2) does not appear in the corresponding bound (7.3) in [6]; the result may be correct without it, but the proof sketched in [6] and detailed here seems to produce it naturally. The logarithm from (2.2) reappears in (2.3), the proof of (2.3) being as for (2.14) in [3, which gives the restriction to $|t|>2 X$. Note that this restriction in range is consistent with $(2.1)$ for large $l$ in the complementary range $|t| \ll X$. These differences make little or no difference to the application here; nonetheless, we provide complete proofs, which we delay until the last paragraph since they are purely analytic and have little to do with the rest of the paper.

In the remainder of this section all notations are as in (1.17) and (1.18), and $r, s$ are coprime integers.

Proposition 2.2. Let $f(x)$ be as in Lemma 2.1 with $k \geq 2$, and $X<1$, let $C=\sqrt{M n} / s \sqrt{r} X$ and

$$
\mathscr{S}_{ \pm}=\sum_{(c, r)=1} \frac{1}{c} \sum_{m \sim M} \alpha_{m} S(m \bar{r}, \pm n ; s c) f\left(\frac{4 \pi \sqrt{m n}}{s \sqrt{r} c}\right) .
$$


Then

$$
\mathscr{S}_{ \pm}=\mathscr{S}_{ \pm}^{\mathrm{exc}}+\mathscr{S}_{ \pm}^{\mathrm{reg}}
$$

where $\mathscr{S}_{ \pm}^{\text {exc }}$ is the contribution from exceptional eigenvalues for $\Gamma_{0}(r s)$, given by

$$
\mathscr{S}_{+}^{\mathrm{exc}}=s \sqrt{r} e\left(-\frac{n \bar{s}}{r}\right) \sum_{\lambda_{j}-\mathrm{exc}}^{\Gamma_{0}(r s)} \frac{\hat{f}\left(t_{j}\right)}{\cosh \pi t_{j}} \sum_{m \sim M} \alpha_{m} \bar{\varrho}_{j \infty}(m) \varrho_{j \frac{1}{s}}(n)
$$

or

$$
\mathscr{S}_{-}^{\mathrm{exc}}=s \sqrt{r} e\left(\frac{n \bar{s}}{r}\right) \sum_{\lambda_{j}-\mathrm{exc}}^{\Gamma_{0}(r s)} \frac{\check{f}\left(t_{j}\right)}{\cosh \pi t_{j}} \sum_{m \sim M} \alpha_{m} \varrho_{j \infty}(m) \varrho_{j \frac{1}{s}}(n)
$$

and $\mathscr{S}_{ \pm}^{\text {reg }}$ is the contribution from the regular spectrum, which can be bounded by

$$
\mathscr{S}_{ \pm}^{\mathrm{reg}} \ll\|\alpha\| s \sqrt{r}\left(Z^{3 / 4}+\sqrt{M / r s}\right)\left(Z^{3 / 4}+\sqrt{n / r s}\right)(M n r s C Z)^{\varepsilon}
$$

for any $\varepsilon>0$.

Proof. The proof is very similar to those of Theorems 8, 9 and 13 in [6]. The key is in recognising that for $\Gamma=\Gamma_{0}(r s)$,

$$
S_{\infty \frac{1}{s}}(m, n ; \gamma)=e\left(\frac{n \bar{s}}{r}\right) S(m \bar{r}, n ; s c)
$$

where the sum on the left is defined for $\gamma=s \sqrt{r} c$ with $c$ coprime to $r$ (see (1.6) in 6]). It follows that for the case $+n$ the sum $(2.6)$ is

$$
\mathscr{S}_{+}=s \sqrt{r} e\left(-\frac{n \bar{s}}{r}\right)\left\{\mathscr{S}_{1}+\mathscr{S}_{2}+\mathscr{S}_{3}\right\}
$$

where $\mathscr{S}_{1}, \mathscr{S}_{2}$ and $\mathscr{S}_{3}$ are the contributions from the three types of spectrum as in (1.17). These are estimated using the bounds from Lemma 2.1 and the corresponding large sieve inequality from (1.20) (as used for similar results in [6]) except that the contribution to $\mathscr{S}_{2}$ from exceptional eigenvalues is separated off, contributing precisely $\mathscr{S}_{+}^{\text {exc }}$ as in the statement. The contribution to $\mathscr{S}_{2}$ from eigenvalues larger than $1 / 4$, denoted by $\mathscr{S}_{2}^{\text {reg }}$, is bounded by splitting the sum at $T_{1}$ and $T_{2}$ such that $T_{2}>Z$ and $1 \leq T_{1}<T_{2}$, and applying (2.1), (2.2) and (2.3) to the corresponding parts. Thus, recalling that $X<1$,

$$
\begin{aligned}
\mathscr{S}_{2}^{\mathrm{reg}} \ll(1+\mid & \log (X / Z) \mid) \sum_{\left|t_{j}\right| \leq T_{1}} \frac{1}{\cosh \pi t_{j}}\left|\sum_{m \sim M} \alpha_{m} \overline{\varrho_{j \infty}(m)}\right|\left|\varrho_{j \frac{1}{s}}(n)\right| \\
+ & Z \sum_{T_{1}<\left|t_{j}\right| \leq T_{2}}\left|t_{j}\right|^{-3 / 2} \frac{1}{\cosh \pi t_{j}}\left|\sum_{m \sim M} \alpha_{m} \overline{\varrho_{j \infty}(m)}\right|\left|\varrho_{j \frac{1}{s}}(n)\right| \\
& +Z^{2} \sum_{\left|t_{j}\right|>T_{2}}\left|t_{j}\right|^{-5 / 2} \frac{1}{\cosh \pi t_{j}}\left|\sum_{m \sim M} \alpha_{m} \overline{\varrho_{j \infty}(m)}\right|\left|\varrho_{j \frac{1}{s}}(n)\right| .
\end{aligned}
$$

In each of these terms we apply Cauchy's inequality and (1.20), and choosing $T_{1}=$ $Z^{3 / 4}, T_{2}=Z$, and noting that $\mu(\infty)=\mu(1 / s)=1 / r s$ gives

$$
\mathscr{S}_{2}^{\mathrm{reg}} \ll\|\alpha\|(1+\log (Z / X))\left(Z^{3 / 2}+\frac{M^{1+\varepsilon}}{r s}\right)^{1 / 2}\left(Z^{3 / 2}+\frac{n^{1+\varepsilon}}{r s}\right)^{1 / 2} .
$$


The contribution from holomorphic forms is

$$
\mathscr{S}_{1}=\frac{1}{\pi} \sum_{\substack{k \equiv 0 \\(\bmod 2)}} \tilde{f}(k-1) \frac{i^{k}(k-1) !}{(4 \pi)^{k-1}} \sum_{j} \psi_{j k}(1 / s, n) \sum_{m \sim M} \alpha_{m} \bar{\psi}_{j k}(\infty, m)
$$

and the contribution from the continuous spectrum is

$$
\mathscr{S}_{3}=\frac{1}{\pi} \sum_{\mathfrak{c}} \int_{-\infty}^{\infty} \hat{f}(t) n^{i t} \varphi_{\mathfrak{c} \frac{1}{s} n}\left(\frac{1}{2}+i t\right) \sum_{m \sim M} \alpha_{m} m^{-i t} \bar{\varphi}_{\mathfrak{c} \infty m}\left(\frac{1}{2}+i t\right) d t .
$$

These are estimated in the same way as $\mathscr{S}_{2}^{\text {reg }}$, substituting the corresponding version of (1.20), which gives (2.7). The case $-n$ is similar, using (1.18) in place of (1.17), and the bounds for $\check{f}$ in place of $\hat{f}$.

Note that we have used (1.20) to bound

$$
\sum_{\left|t_{j}\right| \leq T} \frac{1}{\cosh \pi t_{j}}\left|\varrho_{j \frac{1}{s}}(n)\right|^{2} \ll(1+T)^{2}+\mu(1 / s) n^{1+\varepsilon}=(1+T)^{2}+\frac{n^{1+\varepsilon}}{r s} .
$$

In our application this is more efficient than applying Kim and Sarnak's bound from [18] as in [1] at the corresponding point in the proof of (1.16), since $n$ will be small compared to rs. As remarked above this is not the case in the wider range of uniformity considered in 1 .

Proposition 2.3. Suppose the hypotheses of Proposition 2.2 hold with $X^{2} \leq X_{0}$ and $n / s C^{2} \leq X_{0}$, where $X_{0}$ is as in Lemma 2.1, and $n<r s$. Then

$$
\begin{aligned}
\mathscr{S}_{+}^{\text {exc }} \ll\|\alpha\|\left\{s r^{1 / 2}+s^{1 / 2} M^{1 / 2}+\left(s^{3 / 4}+M^{1 / 4} s^{1 / 2} r^{-1 / 4}\right)\right. & \left.(n, r s)^{1 / 4} C^{1 / 2}\right\} \\
& \times Z^{1 / 2}(r s M C Z)^{\varepsilon}
\end{aligned}
$$

for any $\varepsilon>0$.

Proof. We take $i t_{j}>0$ and introduce a parameter $W \geq 1$, to be optimised later, such that $X W<Z$ and such that $(X W / Z)^{2} \leq X_{0}$. Cauchy's inequality gives

$$
\mathscr{S}_{+}^{\text {exc }} \ll s r^{1 / 2} \sum_{\lambda_{j}-\operatorname{exc}}\left|\hat{f}\left(t_{j}\right)\right|\left|\sum_{m \sim M} \alpha_{m} \bar{\varrho}_{j \infty}(m)\right|\left|\varrho_{j \frac{1}{s}}(n)\right| \ll s r^{1 / 2} A^{1 / 2} B^{1 / 2}
$$

where

$$
A=\sum_{\lambda_{j}-\mathrm{exc}} W^{4 i t_{j}}\left|\sum_{m \sim M} \alpha_{m} \bar{\varrho}_{j \infty}(m)\right|^{2}, B=\sum_{\lambda_{j}-\mathrm{exc}} W^{-4 i t_{j}}\left|\hat{f}\left(t_{j}\right)\right|^{2}\left|\varrho_{j \frac{1}{s}}(n)\right|^{2} .
$$

Applying (1.21),

$$
A \ll\|\alpha\|^{2}(1+W \sqrt{M / r s})\left(1+\sqrt{M^{1+\varepsilon} / r s}\right) .
$$

Note that as observed above this is better than applying (1.20) here.

To estimate $B$ we will use the sum formula (1.17) once more, taking the same group $\Gamma_{0}(r s)$, but with $m=n$ and $\mathfrak{a}=\mathfrak{b}=1 / s$, and with a different test function. Specifically, let $g(x)$ denote a $\mathscr{C}^{\infty}$ function supported on $\left[X_{1}, 2 X_{1}\right]$ and with 
derivatives of all orders bounded by $g^{(\nu)}(x) \ll X_{1}^{-\nu}$, where $X_{1}=(X W / Z)^{2}$. Then (1.17) implies

$$
\sum_{c \equiv 0(\bmod r s)} \frac{1}{c} g\left(\frac{4 \pi n}{c}\right) S_{\frac{1}{s} \frac{1}{s}}(n, n ; c)=\mathscr{S}_{1}+\mathscr{S}_{2}+\mathscr{S}_{3}
$$

where

$$
\begin{aligned}
& \mathscr{S}_{1}=\frac{1}{\pi} \sum_{\substack{k \equiv 0 \\
\bmod 2)}} \sum_{j} \frac{i^{k}(k-1) !}{(4 \pi)^{k-1}} \tilde{g}(k-1)\left|\psi_{j k}\left(\frac{1}{s}, n\right)\right|^{2} \\
& \mathscr{S}_{2}=\sum_{j \geq 1} \frac{\hat{g}\left(t_{j}\right)}{\cosh \pi t_{j}}\left|\varrho_{j \frac{1}{s}}(n)\right|^{2}, \\
& \mathscr{S}_{3}=\frac{1}{\pi} \sum_{\mathfrak{c}} \int_{-\infty}^{\infty}\left|\varphi_{\mathfrak{c} \frac{1}{s} n}\left(\frac{1}{2}+i t\right)\right|^{2} \hat{g}(t) d t .
\end{aligned}
$$

The second expression splits into regular and exceptional parts, denoted by $\mathscr{S}_{2}^{\text {reg }}$ and $\mathscr{S}_{2}^{\text {exc }}$, respectively, where the first contains those terms with real $t_{j}$, and the second contains those with $\lambda_{j}<1 / 4$. From (2.4) we have

$$
f\left(t_{j}\right) \ll(1+\log (Z / X))(Z / X)^{2 i t_{j}},
$$

and can apply (2.5) to obtain

$$
\begin{aligned}
B & \ll(1+\log (Z / X))^{2} \sum_{\lambda_{j}-\operatorname{exc}} X_{1}^{-2 i t_{j}}\left|\varrho_{j \frac{1}{s}}(n)\right|^{2} \\
& \ll(1+\log (Z / X))^{2} \sum_{\lambda_{j}-\operatorname{exc}} \frac{\hat{g}\left(t_{j}\right)}{\cosh \pi t_{j}}\left|\varrho_{j \frac{1}{s}}(n)\right|^{2} \\
& =(1+\log (Z / X))^{2} \mathscr{S}_{2}^{\text {exc }}
\end{aligned}
$$

since $2^{-1 / 2}<\cosh \pi t_{j} \leq 1$, so to estimate $B$ it will be sufficient to estimate $\mathscr{S}_{1}$, $\mathscr{S}_{2}^{\text {reg }}, \mathscr{S}_{3}$, and the sum of Kloosterman sums in (2.9), and absorb the logarithms into the factor $(r s C M C Z)^{\varepsilon}$ in the statement. The estimation of the first three terms is similar to the proof of Proposition 2.2, except that $X_{1}$ is in place of $X$ and 1 is in place of $Z$. Thus, recalling that $X_{1}<X_{0}<1$,

$$
\mathscr{S}_{2}^{\mathrm{reg}}:=\sum_{\lambda_{j} \geq 1 / 4} \frac{\hat{g}\left(t_{j}\right)}{\cosh \pi t_{j}}\left|\varrho_{j \frac{1}{s}}(n)\right|^{2} \ll\left(1+\left|\log X_{1}\right|\right)\left(1+\frac{n^{1+\varepsilon}}{r s}\right)
$$

by applying (2.1) and (2.3) above and below $1+2 X_{1}$. This bound also holds for $\mathscr{S}_{1}$ and $\mathscr{S}_{3}$ by the same reasoning, using the other cases of (1.20). To bound the sum of Kloosterman sums we apply the Weil bound for the Kloosterman sums (see Lemma 2.6 in [6]),

$$
\begin{aligned}
\sum_{\substack{c \equiv 0 \\
(\bmod r s)}} \frac{1}{c} g\left(\frac{4 \pi n}{c}\right) S_{\frac{11}{s} s}(n, n ; c) & \ll \sum_{\substack{c \equiv 0 \\
(\bmod r s)}} g\left(\frac{4 \pi n}{c}\right)(n, c)^{1 / 2} c^{-1 / 2} \tau(c) \\
& \ll \frac{n^{1 / 2}(n, r s)^{1 / 2}}{X_{1}^{1 / 2} r s}\left(n / X_{1}\right)^{\varepsilon}
\end{aligned}
$$


so by $(2.11)$ and (2.12),

$$
B \ll\left(1+\frac{Z n^{1 / 2}(n, r s)^{1 / 2}}{X W r s}\right)(Z n / X W)^{\varepsilon} .
$$

To complete the proof we now consider two cases. If $M<r s$, we choose $W=$ $Z(r s / M)^{1 / 2}$, so $W>1$ and $X_{1}=(X W / Z)^{2}=n / s C^{2} \leq X_{0}$ as required. The other case is $M \geq r s$; we choose $W=Z$, so $W>1$ and $X_{1}=X^{2} \leq X_{0}$. In either case the proposition follows by applying the bounds for $A$ and $B$ in (2.8).

The proof of Theorem 1.6 is now completed by considering the Fourier pair,

$$
F(t, x)=\int_{-\infty}^{\infty} G\left(u, \frac{4 \pi \sqrt{t n}}{s \sqrt{r} x}\right) e(t u) d u, G(u, \xi)=\int_{-\infty}^{\infty} F\left(t, \frac{4 \pi \sqrt{t n}}{s \sqrt{r} \xi}\right) e(-u t) d t,
$$

in a similar manner to 6. If $F$ is as in Theorem 1.6 , then $G(u, \xi)$ is compactly supported on $\xi \asymp X:=\sqrt{M n} / s \sqrt{r} C$ and decays rapidly in $u$ once $u>Z M^{-1+\varepsilon}$. Furthermore,

so

$$
\frac{\partial^{\nu}}{\partial \xi^{\nu}} G(u, \xi) \ll M\left(\frac{Z}{X}\right)^{\nu}, \nu=0,1,2 \ldots
$$

$$
F(m, c)=\int_{|u| \leq Z M^{-1+\varepsilon}} G\left(u, \frac{4 \pi \sqrt{m n}}{s \sqrt{r} c}\right) e(m u) d u+O\left(Z M^{-100}\right) .
$$

In effect this replaces $F(m, c)$ by

$$
f\left(\frac{4 \pi \sqrt{m n}}{s \sqrt{r} c}\right)=G\left(u, \frac{4 \pi \sqrt{m n}}{s \sqrt{r} c}\right)
$$

where $f(x)$ is compactly supported on $x \asymp X$ and $f^{(\nu)}(x) \ll M(Z / X)^{\nu}$. Theorem 1.6 now follows from Propositions 2.2 and 2.3.

\section{A particular Kloosterman sum}

To prepare the ground for later calculations, we must consider a Kloosterman sum with an additional condition on the summands. Given $q>0$, squarefree $d>0$ such that $d^{2} \mid q$, and distinct integers $m_{3}, m_{4}$ such that $\left(m_{3}, m_{4}\right)=1$ and $\left(m_{3} m_{4}, q\right)=1$, the sum to be considered is

$$
\begin{aligned}
& \frac{1}{d} \sum_{u(\bmod q)}^{*} \sum_{v(\bmod d)} e_{q}\left(\left(m_{4}\left(1+v q^{\prime}\right)-m_{3}\right) u-\overline{m_{4} u}\left(1-v q^{\prime}\right) r_{1}+\overline{m_{3} u} r_{2}\right) \\
& =\sum_{\substack{u(\bmod q) \\
m_{4} u+\frac{m_{4} u}{m_{1}} \equiv 0(\bmod d)}}^{*} e_{q}\left(\left(m_{4}-m_{3}\right) u+\overline{m_{3} m_{4}}\left(m_{4} r_{2}-m_{3} r_{1}\right) \bar{u}\right)
\end{aligned}
$$

where $q^{\prime}=q / d$. We will only consider moduli $q$ depending on the greatest common divisor $\left(m_{3}-m_{4}, d\right)$ in a certain way; since our application permits some flexibility in the choice of moduli $q$ this will be sufficient for our purposes.

Lemma 3.1. For each $p_{j}$ dividing d let $p_{j}^{\alpha_{j}} \|\left(m_{3}-m_{4}\right)$ and define

$$
D=\prod p_{j}^{\alpha_{j}+1}=d\left(d^{\infty}, m_{3}-m_{4}\right) .
$$


Then for all $q$ divisible by $D^{2}$ the sum (3.1) equals

$$
S\left(m_{4}-m_{3}, \overline{m_{3} m_{4}}\left(m_{4} r_{2}-m_{3} r_{1}\right) ; q\right)
$$

if $m_{3}^{2} r_{1} \equiv m_{4}^{2} r_{2}$ modulo $D$ and is zero otherwise.

Proof. For each $p_{j}$ write $q=p_{j}^{\beta_{j}} q_{j}$ where $\left(q_{j}, p_{j}\right)=1$, and $q=q_{0} d_{1}$ where $d_{1}=$ $\prod p_{j}^{\beta_{j}}$ and $\left(q_{0}, d\right)=1$. Note that $\beta_{j} \geq 2 \alpha_{j}+2$. The sum (3.1) can be factored as

$$
\begin{aligned}
& S\left(\bar{d}_{1}\left(m_{4}-m_{3}\right), \bar{d}_{1} \overline{m_{3} m_{4}}\left(m_{4} r_{2}-m_{3} r_{1}\right) ; q_{0}\right) \\
& \quad \times \prod_{j} \sum_{\substack{u_{j}\left(\bmod p_{j}^{\beta_{j}}\right) \\
m_{4} u_{j}+\bar{m}_{4} u_{j} r_{1} \equiv 0\left(\bmod p_{j}\right)}}^{*} e\left(\frac{\bar{q}_{j}\left(m_{4}-m_{3}\right) u_{j}}{p^{\beta_{j}}}+\frac{\bar{q}_{j} \overline{m_{3} m_{4}}\left(m_{4} r_{2}-m_{3} r_{1}\right) \bar{u}_{j}}{p^{\beta_{j}}}\right) .
\end{aligned}
$$

We now consider the sum modulo $p_{j}^{\gamma_{j}}$. Dropping the subscript $j$ everywhere, if we define $\gamma=\beta / 2$ or $\gamma=(\beta-1) / 2$ according to whether $\beta$ is even or odd, then

$$
\begin{aligned}
& \sum_{u\left(\bmod p^{\beta}\right)}^{*} e\left(\frac{\bar{q}\left(m_{4}-m_{3}\right) u}{p^{\beta}}+\frac{\overline{q m_{3} m_{4}}\left(m_{4} r_{2}-m_{3} r_{1}\right) \bar{u}}{p^{\beta}}\right) \\
&= \frac{1}{p^{\gamma}} \sum_{u\left(\bmod p^{\beta}\right)}^{*} \sum_{w\left(\bmod p^{\gamma}\right)} e\left(\frac{\bar{q}\left(m_{4}-m_{3}\right) u\left(1+w p^{\beta-\gamma}\right)}{p^{\beta}}\right. \\
&=\left.+\frac{\overline{q m_{3} m_{4}}\left(m_{4} r_{2}-m_{3} r_{1}\right) \bar{u}\left(1-w p^{\beta-\gamma}\right)}{p^{\beta}}\right) \\
& \sum_{\left(m_{4}-m_{3}\right) u-\overline{m_{3} m_{4}}\left(m_{4} r_{2}-m_{3} r_{1}\right) \bar{u} \equiv 0\left(\bmod p^{\gamma}\right)}^{*} e\left(\frac{\bar{q}\left(m_{4}-m_{3}\right) u}{p^{\beta}}\right. \\
&\left.+\frac{\overline{q m_{3} m_{4}}\left(m_{4} r_{2}-m_{3} r_{1}\right) \bar{u}}{p^{\beta}}\right) .
\end{aligned}
$$

In other words, the new congruence condition modulo $p^{\gamma}$ is moot. By the same argument,

$$
\begin{gathered}
\sum_{\substack{u\left(\bmod p^{\beta}\right) \\
m_{4} u+\bar{m}_{4} \bar{u} r_{1} \equiv 0(\bmod p)}}^{*} e\left(\frac{\bar{q}\left(m_{4}-m_{3}\right) u}{p^{\beta}}+\frac{\overline{q m_{3} m_{4}}\left(m_{4} r_{2}-m_{3} r_{1}\right) \bar{u}}{p^{\beta}}\right) \\
=\sum_{\substack{u\left(\bmod p^{\beta}\right) \\
(3.3)}}^{*} e\left(\frac{\bar{q}\left(m_{4}-m_{3}\right) u}{p^{\beta}}+\frac{\overline{q m_{3} m_{4}}\left(m_{4} r_{2}-m_{3} r_{1}\right) \bar{u}}{p^{\beta}}\right) . \\
\left(m_{4}-m_{3}\right) u-\bar{m}_{3} m_{4}\left(m_{4} r_{2}-m_{3} r_{1}\right) \bar{u} \equiv 0\left(\bmod p^{\gamma}\right)
\end{gathered}
$$

The condition modulo $p^{\gamma}$ is equivalent to

$$
\left(m_{4}-m_{3}\right) m_{3} m_{4}\left(m_{4} u+\bar{m}_{4} \bar{u} r_{1}\right)+\left(m_{3}^{2} r_{1}-m_{4}^{2} r_{2}\right) \bar{u} \equiv 0\left(\bmod p^{\gamma}\right),
$$

so recalling that $p^{\alpha} \|\left(m_{3}-m_{4}\right)$ and that $\gamma \geq \alpha+1$, the condition modulo $p$ is equivalent to the requirement that $p^{\alpha+1}$ divides $m_{3}^{2} r_{1}-m_{4}^{2} r_{2}$. (Note also that if the sum is non-vanishing, then the summands satisfy $m_{3} u+\bar{m}_{3} \bar{u} r_{2} \equiv 0$ modulo $p$, as one 
would expect.) Summarising, the sum vanishes unless $p^{\alpha+1}$ divides $m_{3}^{2} r_{1}-m_{4}^{2} r_{2}$, in which case the congruence modulo $p$ can be dropped, and the congruence modulo $p^{\gamma}$ can be dropped also, by (3.2). Reintroducing the subscripts $j$, the proof is completed by closing the product over $j$.

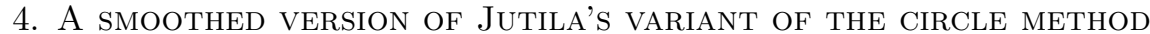

Let $\mathscr{Q} \subseteq[Q, 2 Q]$ for some $Q>1$ be a prescribed set of moduli $q$ and let

$$
L=\sum_{q \in \mathscr{Q}} \omega(q) \sum_{u(\bmod q)}^{*} 1
$$

denote the weighted total number of reduced fractions modulo $q$ for $q \in \mathscr{Q}$. Let $\phi$ be even and supported on $[-1,1]$ with $\int \phi=1$, let $\phi_{\delta}(x)=\phi(x / \delta)$ for any $\delta>0$, and let $J_{\delta}(\alpha)$ denote the periodic function

$$
J_{\delta}(\alpha)=\frac{1}{\delta L} \sum_{q \in \mathscr{Q}} \omega(q) \sum_{u(\bmod q)}^{*} \sum_{n \in \mathbb{Z}} \phi_{\delta}\left(\frac{u}{q}+\alpha+n\right) .
$$

In 14 and 15 Jutila considers essentially the same function, although defined slightly differently and with $\phi_{\delta}$ given by the characteristic function of the interval $[-\delta, \delta]$, and shows that

$$
\int_{0}^{1}\left|1-J_{\delta}(\alpha)\right|^{2} d \alpha \ll \frac{Q^{2+\varepsilon}}{L^{2} \delta}
$$

for $Q^{-2} \ll \delta \ll Q^{-1}$. We will prove a version where $\phi \in \mathscr{C}^{\infty}$, referring the reader to 14 and 15 for the unsmoothed case. (See also [1] and 12]).

The Fourier expansion of $J_{\delta}$ can be calculated as

$$
J_{\delta}(\alpha)=\sum_{m=-\infty}^{\infty} e(m \alpha) \int_{0}^{1} J_{\delta}(x) e(-m x) d x
$$

where

$$
\begin{aligned}
\int_{0}^{1} J_{\delta}(x) e(-m x) d x & =\frac{1}{\delta L} \sum_{q \in \mathscr{Q}} \omega(q) \sum_{u(\bmod q)}^{*} \int_{-\infty}^{\infty} \phi_{\delta}\left(\frac{u}{q}+x\right) e(-m x) d x \\
& =\frac{1}{L} \sum_{q \in \mathscr{Q}} \omega(q) \sum_{u(\bmod q)}^{*} \hat{\phi}(\delta m) e_{q}(u m)
\end{aligned}
$$

and $\hat{\phi}$ is the Fourier transform, bounded by

$$
\hat{\phi}(\xi)=\int_{-\infty}^{\infty} \phi(x) e(-x \xi) d x \ll(1+|\xi|)^{-A}
$$

for any $A>0$, by repeated integration by parts. Noting that $\hat{\phi}(0)=1$,

$$
\int_{0}^{1}\left|1-J_{\delta}(\alpha)\right|^{2} d \alpha=\frac{1}{L^{2}} \sum_{m \neq 0}|\hat{\phi}(\delta m)|^{2}\left|\sum_{q \in \mathscr{Q}} \omega(q) \sum_{u(\bmod q)}^{*} e_{q}(u m)\right|^{2}
$$


by Parseval's formula. The sum over $u$ is a Ramanujan sum and is equal to $\sum_{d \mid(m, q)} \mu(q / d) d$, so bounding $\hat{\phi}$ using (4.2) we obtain

$$
\int_{0}^{1}\left|1-J_{\delta}(\alpha)\right|^{2} d \alpha \ll \frac{1}{L^{2}} \sum_{m \geq 1}(1+\delta m)^{-A}\left|\sum_{Q \leq q \leq 2 Q} \sum_{d \mid(m, q)} d\right|^{2},
$$

which implies (4.1).

\section{Analysis of the shifted COnvolution}

We first prove Theorem 1.4, recalling that $f(z)$ and $g(z)$ are two cusp forms of weight $k$ for the modular group, that $m_{1}, m_{2}$ are two distinct squarefree integers with $d=\left(m_{1}, m_{2}\right)$ and that $D=\left(m_{1}-m_{2}, d^{\infty}\right)$. Without loss of generality, we can suppose that $m_{1} m_{2}<N$, since the result is trivial if $m_{1} m_{2} \geq N$. Writing $m_{1}=d m_{3}, m_{2}=d m_{4}$, and introducing the new variables $k_{1}=d m_{3} n-1$ and $k_{2}=d m_{4} n-1$, the sum to be bounded is

$$
\begin{gathered}
\mathscr{C}=\sum_{n=1}^{\infty} e^{-4 \pi n / N} A\left(d m_{3} n-1\right) \overline{B\left(d m_{4} n-1\right)} \\
=e^{-2 \pi / m_{1} N-2 \pi / m_{2} N} \sum_{\substack{k_{1}, k_{2}=1 \\
k_{1} \equiv-1(\bmod d) \\
m_{4}\left(k_{1}+1\right)=m_{3}\left(k_{2}+1\right)}}^{\infty} A\left(k_{1}\right) \overline{B\left(k_{2}\right)} e^{-2 \pi\left(k_{1} / m_{1} N+k_{2} / m_{2} N\right)} .
\end{gathered}
$$

From the expansions

we have

$$
\begin{gathered}
f\left(m_{4} \alpha+\frac{v}{d}+\frac{i}{m_{1} N}\right)=\sum_{k=1}^{\infty} A(k) e\left(k m_{4} \alpha\right) e_{d}(k v) e^{-2 \pi k / m_{1} N}, \\
g\left(m_{3} \alpha+\frac{i}{m_{2} N}\right)=\sum_{k=1}^{\infty} B(k) e\left(k m_{3} \alpha\right) e^{-2 \pi k / m_{2} N}
\end{gathered}
$$

$$
\begin{aligned}
\int_{0}^{1} e\left(\left(m_{4}-m_{3}\right) \alpha\right) f\left(m_{4} \alpha+\frac{v}{d}+\frac{i}{m_{1} N}\right) \bar{g}\left(m_{3} \alpha+\frac{i}{m_{2} N}\right) d \alpha \\
=\sum_{\substack{k_{1}, k_{2}=1 \\
m_{4}\left(k_{1}+1\right)=m_{3}\left(k_{2}+1\right)}}^{\infty} A\left(k_{1}\right) \overline{B\left(k_{2}\right)} e_{d}\left(k_{1} v\right) e^{-2 \pi\left(k_{1} / m_{1} N+k_{2} / m_{2} N\right)}
\end{aligned}
$$

and hence

$$
\mathscr{C}=\frac{\gamma}{d} \sum_{v(\bmod d)} e_{d}(v) \int_{0}^{1} e\left(\left(m_{4}-m_{3}\right) \alpha\right) f\left(m_{4} \alpha+\frac{v}{d}+\frac{i}{K_{1}}\right) \bar{g}\left(m_{3} \alpha+\frac{i}{K_{2}}\right) d \alpha .
$$

where $K_{1}=d m_{3} N, K_{2}=d m_{4} N$ and $\gamma=e^{-2 \pi / m_{1} N-2 \pi / m_{2} N}$.

We now apply the method from Section 4 , taking $\phi$ to be smooth, and taking $\omega(q)$ to be $\mathscr{C}^{\infty}$ on $(0, \infty)$, zero outside $[Q, 2 Q], 1$ in $[4 Q / 3,5 Q / 3]$ and with derivatives of all orders bounded by

$$
\omega^{(\nu)}(x) \ll Q^{-\nu}, \nu=0,1,2 \ldots
$$


We choose $\mathscr{Q}$ to consist of those $q$ in $[Q, 2 Q]$ which are divisible by $D^{2}$ and coprime to $m_{3} m_{4}$. For the present, $Q$ is left undefined, but will later be taken as a sufficiently large power of $m_{1} m_{2} N$. We also define $\delta=Q^{-1}$. Note that

$$
Q^{2} D^{-2}\left(D m_{3} m_{4}\right)^{-\varepsilon} \ll L \ll Q^{2} D^{-2}
$$

for any $\varepsilon>0$. We consider

$$
\mathscr{C}_{1}=\frac{\gamma}{d} \sum_{v(\bmod d)} e_{d}(v) \int_{0}^{1} e\left(\left(m_{4}-m_{3}\right) \alpha\right) f\left(m_{4} \alpha+\frac{v}{d}+\frac{i}{K_{1}}\right) \bar{g}\left(m_{3} \alpha+\frac{i}{K_{2}}\right) J_{\delta}(\alpha) d \alpha
$$

as an approximation to $\mathscr{C}$. By the Cauchy-Schwarz inequality

$$
\begin{aligned}
\left|\mathscr{C}-\mathscr{C}_{1}\right| \leq\left\{\int_{0}^{1}\left|f\left(m_{4} \alpha+\frac{v}{d}+\frac{i}{K_{1}}\right)\right|^{2}\left|g\left(m_{3} \alpha+\frac{i}{K_{2}}\right)\right|^{2} d \alpha\right\}^{1 / 2} & \\
& \times\left\{\int_{0}^{1}\left|1-J_{\delta}(\alpha)\right|^{2} d \alpha\right\}^{1 / 2}
\end{aligned}
$$

and applying the standard bounds $f(z), g(z) \ll y^{-k / 2}$ in the first factor and (4.1) in the second, we obtain

$$
\mathscr{C}=\mathscr{C}_{1}+O\left(\left(K_{1} K_{2}\right)^{k / 2} Q^{1+\varepsilon} L^{-1} \delta^{-1 / 2}\right) .
$$

Expanding $J_{\delta}$ we can use the sum over $n \in \mathbb{Z}$ to complete the range of integration to $(-\infty, \infty)$, so

$$
\begin{aligned}
\mathscr{C}_{1}= & \frac{\gamma}{L d \delta} \sum_{q \in \mathscr{Q}} \omega(q) \sum_{u(\bmod q)}^{*} \sum_{v(\bmod d)} e_{d}(v) \\
& \times \int_{-\infty}^{\infty} e\left(\left(m_{4}-m_{3}\right) \alpha\right) f\left(m_{4} \alpha+\frac{v}{d}+\frac{i}{K_{1}}\right) \bar{g}\left(m_{3} \alpha+\frac{i}{K_{2}}\right) \phi_{\delta}\left(\frac{u}{q}+\alpha\right) d \alpha
\end{aligned}
$$

and shifting the variable $\alpha$,

$$
\begin{aligned}
& \mathscr{C}_{1}= \frac{\gamma}{L d \delta} \sum_{q \in \mathscr{Q}} \omega(q) \sum_{u(\bmod q)}^{*} e_{q}\left(\left(m_{4}-m_{3}\right) u\right) \sum_{v(\bmod d)} e_{d}(v) \\
& \times \int_{-\infty}^{\infty} e\left(\left(m_{4}-m_{3}\right) \alpha\right) f\left(m_{4} \alpha-\frac{m_{4} u}{q}+\frac{v}{d}+\frac{i}{K_{1}}\right) \\
& \times \bar{g}\left(m_{3} \alpha-\frac{m_{3} u}{q}+\frac{i}{K_{2}}\right) \phi_{\delta}(\alpha) d \alpha .
\end{aligned}
$$

While the expression $e_{q}\left(\left(m_{4}-m_{3}\right) u\right)$ plays a vital role, the corresponding factor in the integrand can be approximated by

$$
e\left(\left(m_{4}-m_{3}\right) \alpha\right)=1+O\left(\left|m_{4}-m_{3}\right||\alpha|\right)
$$


where the error is small since $|\alpha|<\delta$, and defining

$$
\begin{aligned}
\mathscr{C}_{2}=\frac{\gamma}{L d \delta} \sum_{q \in \mathscr{Q}} \omega & (q) \sum_{u(\bmod q)}^{*} e_{q}\left(\left(m_{4}-m_{3}\right) u\right) \sum_{v(\bmod d)} e_{d}(v) \\
& \times \int_{-\infty}^{\infty} f\left(m_{4} \alpha-\frac{m_{4} u}{q}+\frac{v}{d}+\frac{i}{K_{1}}\right) \bar{g}\left(m_{3} \alpha-\frac{m_{3} u}{q}+\frac{i}{K_{2}}\right) \phi_{\delta}(\alpha) d \alpha
\end{aligned}
$$

we have

$$
\begin{array}{r}
\left|\mathscr{C}_{1}-\mathscr{C}_{2}\right| \leq \frac{\gamma\left|m_{3}-m_{4}\right|}{\delta} \int_{-\infty}^{\infty}\left|f\left(m_{4} \alpha-\frac{m_{4} u}{q}+\frac{v}{d}+\frac{i}{K_{1}}\right)\right| \\
\times\left|g\left(m_{3} \alpha-\frac{m_{3} u}{q}+\frac{i}{K_{2}}\right)\right|\left|\phi_{\delta}(\alpha)\right| \alpha d \alpha
\end{array}
$$

and hence

$$
\mathscr{C}_{1}=\mathscr{C}_{2}+O\left(\left(K_{1} K_{2}\right)^{k / 2}\left|m_{4}-m_{3}\right| \delta\right)
$$

by again applying the standard bounds $f(z), g(z) \ll y^{-k / 2}$. We now apply variable changes to obtain

$$
\begin{aligned}
\mathscr{C}_{2}=\frac{R \gamma}{L d} \sum_{q \in \mathscr{Q}} \omega(q) & \sum_{u(\bmod q)}^{*} \sum_{v(\bmod d)} e_{q}\left(\left(m_{4}\left(1+v q^{\prime}\right)-m_{3}\right) u\right) \\
& \times \int_{-\infty}^{\infty} \phi(R \xi) f\left(\frac{m_{4} u\left(1+v q^{\prime}\right)}{q}+\frac{\xi+i}{K_{1}}\right) \bar{g}\left(\frac{m_{3} u}{q}+\frac{\xi+i}{K_{2}}\right) d \xi
\end{aligned}
$$

where $q=d q^{\prime}$ as in Section 3 above, and we have introduced $R=Q / d m_{3} m_{4} N$ to simplify notation. Note that were we to apply the standard upper bounds $f(z), g(z) \ll y^{-k / 2}$ at this point, then we would obtain

$$
\mathscr{C}_{2} \ll\left(K_{1} K_{2}\right)^{k / 2} \ll\left(d^{2} m_{3} m_{4} N^{2}\right)^{k / 2} .
$$

The modularity relation for $g$ gives

$$
\begin{aligned}
(q z)^{k} g\left(\frac{m_{3} u}{q}+z\right) & =g\left(\left(\begin{array}{cc}
\overline{m_{3} u} & \left(1-m_{3} u \overline{m_{3} u}\right) / q \\
-q & m_{3} u
\end{array}\right)\left(\frac{m_{3} u}{q}+z\right)\right) \\
& =g\left(-\frac{\overline{m_{3} u}}{q}-\frac{1}{q^{2} z}\right)
\end{aligned}
$$

for any $z \in \mathscr{H}$, and similarly

$$
(q z)^{k} f\left(\frac{m_{4} u\left(1+v q^{\prime}\right)}{q}+z\right)=f\left(-\frac{\overline{m_{4} u}\left(1-v q^{\prime}\right)}{q}-\frac{1}{q^{2} z}\right) .
$$


Applying these relations

$$
\begin{aligned}
\mathscr{C}_{2}= & \frac{R \gamma\left(K_{1} K_{2}\right)^{k}}{L d} \sum_{q \in \mathscr{Q}} \frac{\omega(q)}{q^{2 k}} \sum_{u(\bmod q)}^{*} \sum_{v(\bmod d)} e_{q}\left(\left(m_{4}\left(1+v q^{\prime}\right)-m_{3}\right) u\right) \\
& \times \int_{-\infty}^{\infty} \phi(R \xi) f\left(-\frac{\bar{m}_{4} \bar{u}\left(1-v q^{\prime}\right)}{q}-\frac{K_{1}}{q^{2}(\xi+i)}\right) \bar{g}\left(-\frac{\bar{m}_{3} \bar{u}}{q}-\frac{K_{2}}{q^{2}(\xi+i)}\right) \frac{d \xi}{\left(\xi^{2}+1\right)^{k}} .
\end{aligned}
$$

Note that if we were to apply the bounds $f(z), g(z) \ll y^{-k / 2}$ we would again obtain (5.4). Expanding the cusp forms and applying Lemma 3.1, which is possible since all $q$ are divisible by $D^{2}$, we obtain

$$
\begin{aligned}
\mathscr{C}_{2}= & \frac{R \gamma}{L}\left(K_{1} K_{2}\right)^{k} \sum_{\substack{r_{1}, r_{2}=1 \\
m_{3}^{2} r_{1} \equiv m_{4}^{2} r_{2} \\
(\bmod D)}}^{\infty} A\left(r_{1}\right) \overline{B\left(r_{2}\right)} \\
& \times \sum_{\substack{q \in \mathscr{Q} \\
q^{2 k}}} \frac{\omega(q)}{\left(\bar{m}_{3} \bar{m}_{4}\left(m_{4} r_{2}-m_{3} r_{1}\right), m_{4}-m_{3} ; q\right)} \\
& \times \int_{-\infty}^{\infty} \phi(R \xi) e\left(\frac{d N\left(m_{4} r_{2}-m_{3} r_{1}\right) \xi}{q^{2}\left(\xi^{2}+1\right)}\right) \exp \left(-\frac{2 \pi\left(K_{1} r_{1}+K_{2} r_{2}\right)}{q^{2}\left(\xi^{2}+1\right)}\right) \frac{d \xi}{\left(\xi^{2}+1\right)^{k}} .
\end{aligned}
$$

Defining $R_{1}:=Q^{2} N^{\varepsilon} / K_{1}$ and $R_{2}:=Q^{2} N^{\varepsilon} / K_{2}$ and supposing $R>1 / 2$, which is clearly satisfied for $Q$ sufficiently large, the contribution to $\mathscr{C}_{2}$ from $r_{1}>R_{1}$ is no more than

$$
\begin{aligned}
R \gamma\left(K_{1} K_{2}\right)^{k} Q^{-2 k} \sum_{r_{1}>R_{1}} \sum_{r_{2}=1}^{\infty}\left|A\left(r_{1}\right)\right|\left|B\left(r_{2}\right)\right| \exp ( & \left.-\frac{\pi\left(K_{1} r_{1}+K_{2} r_{2}\right)}{4 Q^{2}}\right) \int_{-\infty}^{\infty} \frac{|\phi(R \xi)| d \xi}{\left(\xi^{2}+1\right)^{k}} \\
& \ll Q^{2+\varepsilon}\left(K_{1} K_{2}\right)^{(k-1) / 2+\varepsilon} e^{-\pi N^{\varepsilon} / 4}
\end{aligned}
$$

by estimating the Kloosterman sum trivially as $O(\phi(q))$ and applying Deligne's bound for the Fourier coefficients, which in turn is no more than $O\left(N^{-100}\right)$, say. A similar argument applies to the sum over $r_{2}$, so it will be sufficient to consider only the ranges $r_{1} \leq R_{1}$ and $r_{2} \leq R_{2}$.

In this range we group the summands by the values $v:=m_{4} r_{2}-m_{3} r_{1}$, treating the special case $v=0$ separately before proceeding. Since $\left(m_{3}, m_{4}\right)=1$, if $m_{4} r_{2}=$ $m_{3} r_{1}$, then $r_{1}=m_{4} r$ and $r_{2}=m_{3} r$ for some $r \in \mathbb{N}$ with $r \leq R_{0}:=Q^{2} N^{\varepsilon} / d m_{3} m_{4} N$, so the contribution from $v=0$ is no more than

$$
\begin{aligned}
\frac{R \gamma}{L}\left(K_{1} K_{2}\right)^{k} & \sum_{r \leq R_{0}}\left|A\left(m_{4} r\right)\right|\left|B\left(m_{3} r\right)\right| \sum_{q \in \mathscr{Q}} \frac{\omega(q)}{q^{2 k}}\left|S\left(0, m_{4}-m_{3} ; q\right)\right| \\
& \times \int_{-\infty}^{\infty} \frac{|\phi(R \xi)|}{\left(\xi^{2}+1\right)^{k}} \exp \left(-\frac{4 \pi d N m_{3} m_{4} r}{q^{2}\left(\xi^{2}+1\right)}\right) d \xi \ll D^{4}\left(K_{1} K_{2}\right)^{(k+1) / 2} N^{\varepsilon} Q^{-1}
\end{aligned}
$$

where we have applied Deligne's bound for the Fourier coefficients and evaluated the Ramanujan sum. Thus

$$
\mathscr{C}_{2}=\mathscr{C}_{3}+O\left(D^{4}\left(K_{1} K_{2}\right)^{(k+1) / 2} N^{\varepsilon} Q^{-1}+Q^{2+\varepsilon}\left(K_{1} K_{2}\right)^{(k-1) / 2+\varepsilon} e^{-\pi N^{\varepsilon} / 4}\right)
$$


where

$$
\begin{aligned}
\mathscr{C}_{3}= & \frac{R \gamma}{L}\left(K_{1} K_{2}\right)^{k} \sum_{\substack{r_{1} \leq R_{1}, r_{2} \leq R_{2} \\
m_{3}^{2} r_{1} \equiv m_{4}^{2} r_{2}(\bmod D) \\
m_{4} r_{2} \neq m_{3} r_{1}}} A\left(r_{1}\right) \overline{B\left(r_{2}\right)} \\
& \times \sum_{\substack{q \in \mathscr{Q} \\
q^{2 k}}} \frac{\omega(q)}{\left(\bar{m}_{3} \bar{m}_{4}\left(m_{4} r_{2}-m_{3} r_{1}\right), m_{4}-m_{3} ; q\right)} \\
& \times \int_{-\infty}^{\infty} \phi(R \xi) e\left(\frac{d N\left(m_{4} r_{2}-m_{3} r_{1}\right) \xi}{q^{2}\left(\xi^{2}+1\right)}\right) \exp \left(-\frac{2 \pi\left(K_{1} r_{1}+K_{2} r_{2}\right)}{q^{2}\left(\xi^{2}+1\right)}\right) \frac{d \xi}{\left(\xi^{2}+1\right)^{k}} .
\end{aligned}
$$

A simple contour integral argument shows that

$$
e^{-2 \pi x}=\frac{1}{\pi} \int_{-\infty}^{\infty} \frac{e(x y) d y}{1+y^{2}}
$$

which we apply for $x=\left(K_{1} r_{1}+K_{2} r_{2}\right) / q^{2}\left(\xi^{2}+1\right)$ followed by a variable change to separate $r_{1}$ and $r_{2}$ from $q$. This gives

$$
\mathscr{C}_{3}=\frac{R \gamma\left(K_{1} K_{2}\right)^{k}}{\pi L} \int_{-\infty}^{\infty} \frac{\phi(R \xi)}{\left(\xi^{2}+1\right)^{k}} \int_{-\infty}^{\infty} \frac{d N q^{2}\left(\xi^{2}+1\right)}{d^{2} N^{2}+q^{4}\left(\xi^{2}+1\right)^{2} y^{2}} \mathscr{C}_{4}(\xi, y) d y d \xi
$$

for

$$
\mathscr{C}_{4}(\xi, y)=\sum_{0<|v| \leq V} \gamma_{v} \sum_{q \in \mathscr{Q}} \frac{\omega(q)}{q^{2 k}} e\left(\frac{d N \nu \xi}{q^{2}\left(\xi^{2}+1\right)}\right) S\left(\bar{m}_{3} \bar{m}_{4} \nu, m_{4}-m_{3} ; q\right)
$$

where

$$
\gamma_{\nu}=\sum_{\substack{r_{1} \leq R_{1}, r_{2} \leq R_{2} \\ m_{4} r_{2}-m_{3} r_{1}=v \\ m_{3}^{2} r_{1} \equiv m_{4}^{2} r_{2}(\bmod D)}} A\left(r_{1}\right) e\left(m_{3} r_{1} y\right) \overline{B\left(r_{2}\right)} e\left(m_{4} r_{2} y\right)
$$

and $V=Q^{2} N^{\varepsilon} / d N$. We continue with the positive values of $v$; the change of sign corresponding to the \pm alternative in Theorem 1.6, so the negative values can be treated in the same way.

Lemma 5.1. With all notation as above, for any $\varepsilon>0$ we have the upper bound

$$
\|\gamma\|^{2}=\sum_{v \leq V}\left|\gamma_{v}\right|^{2} \ll\left(R_{1} R_{2}\right)^{k} \log ^{2} R_{1} \log ^{2} R_{2}
$$

where the implied constant depends only on $\varepsilon$.

Proof. This is similar to Lemma 3 in [15] or Lemma 3.2 in [1]. Opening the square and summing over $v$, we have

$$
\begin{aligned}
& \|\gamma\|^{2}= \\
& \sum_{r_{1}, r_{3} \leq R_{1}, r_{2}, r_{4} \leq R_{2}} \\
& m_{4} r_{2}-m_{3} r_{1}=m_{4} r_{4}-m_{3} r_{1} \\
& m_{3}^{2} r_{1} \equiv m_{4}^{2} r_{2}, m_{3}^{2} r_{3} \equiv m_{4}^{2} r_{4}(\bmod D) \\
& A\left(r_{1}\right) e\left(m_{3} r_{1} y\right) \overline{B\left(r_{2}\right)} e\left(m_{4} r_{2} y\right) \\
& \times \overline{A\left(r_{3}\right)} e\left(-m_{3} r_{3} y\right) B\left(r_{4}\right) e\left(-m_{4} r_{4} y\right) .
\end{aligned}
$$


We can use additive characters to detect the congruences modulo $D$, and an integral over $[0,1]$ to detect the equality, so

$$
\begin{aligned}
&\|\gamma\|^{2}=\frac{1}{D^{2}} \sum_{\substack{u_{1}, u_{2} \\
(\bmod D)}} \int_{0}^{1} \sum_{r_{1}, r_{3} \leq R_{1}} A\left(r_{1}\right) e\left(m_{3} r_{1}(\alpha+y)\right) e_{D}\left(u_{1} m_{3}^{2} r_{1}\right) \\
& \times \overline{A\left(r_{3}\right)} e\left(-m_{3} r_{3}(\alpha+y)\right) e_{D}\left(-u_{2} m_{3}^{2} r_{3}\right) \\
& \times \sum_{r_{2}, r_{4} \leq R_{2}} \overline{B\left(r_{2}\right)} e\left(-m_{4} r_{2}(\alpha-y)\right) e_{D}\left(-u_{1} m_{4}^{2} r_{2}\right) \\
& \times B\left(r_{4}\right) e\left(-m_{4} r_{4}(\alpha-y)\right) e_{D}\left(u_{2} m_{4}^{2} r_{4}\right) d \alpha .
\end{aligned}
$$

A well-known bound due to Wilton gives

$$
\sum_{n \leq N} A(n) e(x n) \ll N^{k / 2} \log N
$$

uniformly in $x \in \mathbb{R}$, so each of the sums over $r_{1}$ and $r_{3}$ is $O\left(R_{1}^{1 / 2} \log R_{1}\right)$ and each of the sums over $r_{2}$ and $r_{4}$ is $O\left(R_{2}^{1 / 2} \log R_{2}\right)$, and the lemma follows.

Introducing the notation

$$
\Omega(\nu, q)=\omega(q)\left(\frac{Q}{q}\right)^{2 k-1} e\left(\frac{d N v \xi}{q^{2}\left(\xi^{2}+1\right)}\right)
$$

we can write

$$
\mathscr{C}_{4}(\xi, y)=Q^{-2 k+1} \sum_{|v| \leq V} \gamma_{v} \sum_{\left(c, m_{3} m_{4}\right)=1} \frac{\Omega\left(\nu, D^{2} c\right)}{D^{2} c} S\left(\bar{m}_{3} \bar{m}_{4} v, m_{4}-m_{3} ; D^{2} c\right) .
$$

Noting that $|\xi| \leq R^{-1}$ we can apply Theorem 1.6 with $M, n, C, Z, r$ and $s$ given by $V, m_{4}-m_{3}, Q D^{-2}, 1, m_{3} m_{4}$ and $D^{2}$, which gives

$$
\begin{aligned}
\mathscr{C}_{4}(\xi, y) \ll \frac{\|\gamma\| Q^{\varepsilon}}{D^{2} Q^{2 k-1}}\left\{D^{2}\left(m_{3} m_{4}\right)^{1 / 2}+D V^{1 / 2}+D^{3 / 4} d^{-1 / 4} Q^{1 / 2}\right. \\
\left.+(D V)^{1 / 4}\left(d m_{3} m_{4}\right)^{-1 / 4} Q^{1 / 2}\right\} .
\end{aligned}
$$

Note that the greatest common divisor $(n, r s)$ from Theorem 1.6 is here equal to $\left(m_{4}-m_{3}, D^{2}\right)=D / d$. Furthermore, the condition $M n / r s^{2} C^{2}<X_{0}$ required for Theorem 1.6 is satisfied for sufficiently large $N$ since

$$
\frac{V\left(m_{4}-m_{3}\right)}{m_{3} m_{4} Q^{2}}=\frac{\left(m_{2}-m_{1}\right)}{m_{1} m_{2}} N^{-1+\varepsilon}<N^{-1+\varepsilon},
$$

and the condition $n / s C^{2}<X_{0}$ is also satisfied since here $n / s C^{2}=\left(m_{4}-m_{3}\right) D^{2} / Q^{2}$, which will be smaller than $X_{0}$ for $Q$ sufficiently large. Applying Lemma 5.1 this implies

$$
\begin{aligned}
\mathscr{C}_{3} \ll \frac{\left(d^{2} m_{3} m_{4} N^{2}\right)^{k / 2+\varepsilon}}{R d m_{3} m_{4} N}\left\{D^{2}\left(m_{3} m_{4}\right)^{1 / 2}+\right. & D R m_{3} m_{4}(d N)^{1 / 2} \\
& \left.+(D / d)^{1 / 4} R\left(d m_{3} m_{4} N\right)^{3 / 4}\right\} Q^{\varepsilon} .
\end{aligned}
$$

Whatever the value of $Q$, hence $R$, the second and third terms provide the error term in the statement of Theorem 1.4. Since $D$ is at most $\left|m_{2}-m_{1}\right|$, which is certainly no more than $2 m_{1} m_{2}$, the first term is $O\left(Q^{-1}\left(m_{1} m_{2}\right)^{(k+5) / 2+\varepsilon} N^{k+\varepsilon}\right)$, and choosing $Q$ to be a sufficiently large power of $m_{1} m_{2} N$, say $Q=\left(m_{1} m_{2} N\right)^{2 k+4}$, 
this is $O\left(\left(m_{1} m_{2} N\right)^{-1}\right)$ and can be dropped. The same applies to the error terms in (5.2), (5.3) and (5.5). The proof of Theorem 1.4 is now complete.

We now sketch a proof of Theorem 1.5. Using (4.1) as above and applying (5.6) in the error term,

$$
\begin{array}{r}
\mathscr{D}_{G}\left(m_{1}, m_{2}, h\right)=\int_{0}^{1} \sum_{k_{1}, k_{2}} G\left(k_{1}, k_{2}\right) a\left(k_{1}\right) \overline{b\left(k_{2}\right)} e\left(\left(m_{1} k_{1}-m_{2} k_{2}-h\right) \alpha\right) J_{\delta}(\alpha) d \alpha \\
+O\left(Z_{1} Z_{2}\left(X_{1} X_{2}\right)^{1 / 2} Q^{1+\varepsilon} L^{-1} \delta^{-1 / 2}\right) .
\end{array}
$$

Here we will choose $\mathscr{Q}$ to be composed of those $q$ in $[Q, 2 Q]$ which are coprime to $m_{1} m_{2}$. Opening $J_{\delta}$ and applying a variable change the remaining sum is

$$
\begin{aligned}
\mathscr{D}_{1}=\frac{1}{2 L} \int_{-\infty}^{\infty} \phi(\xi) & \sum_{q \in \mathscr{Q}} \omega(q) \sum_{u(\bmod q)}^{*} e_{q}(h u) \\
& \times \sum_{k_{1}, k_{2}} G_{\xi}\left(k_{1}, k_{2}\right) a\left(k_{1}\right) e_{q}\left(-u m_{1} k_{1}\right) \overline{b\left(k_{2}\right)} e_{q}\left(u m_{2} k_{2}\right) d \xi \\
& +O\left(Z_{1} Z_{2}\left(X_{1} X_{2}\right)^{1 / 2+\varepsilon}|h| \delta\right)
\end{aligned}
$$

where $G_{\xi}\left(x_{1}, x_{2}\right)=G\left(x_{1}, x_{2}\right) e\left(\left(m_{1} x_{1}-m_{2} x_{2}\right) \delta \xi\right)$ and where the error term has come from dropping the exponential $e(-h \delta \xi)$ as being very close to 1 , again using (5.6).

Instead of using the modularity relations directly as in the proof of Theorem 1.4, we apply them in the form of the well-known Voronoi-type summation formula

$$
\sum_{n} G(n) a(n) e_{q}(u n)=\frac{2 \pi i^{k}}{q} \sum_{m=1}^{\infty} a(m) e_{q}(-\bar{u} m) \int_{0}^{\infty} G(t) J_{k-1}\left(\frac{4 \pi \sqrt{m t}}{q}\right) d t
$$

for smooth compactly supported test functions $G$ and $(u, q)=1$, where $J_{k-1}$ denotes the standard Bessel function. Applying this in $k_{1}$ and $k_{2}$, the sum above is

$$
\begin{aligned}
\mathscr{D}_{1}=\frac{1}{2 L} \int_{-\infty}^{\infty} \phi(\xi) & \sum_{r_{1}, r_{2}} a\left(r_{1}\right) \overline{b\left(r_{2}\right)} \\
& \times \sum_{q \in \mathscr{Q}} \omega(q) H\left(r_{1}, r_{2}, q, \xi\right) S\left(\bar{m}_{1} \bar{m}_{2}\left(m_{2} r_{1}-m_{1} r_{2}\right), h ; q\right) d \xi \\
& +O\left(Z_{1} Z_{2}\left(X_{1} X_{2}\right)^{1 / 2+\varepsilon}|h| \delta\right)
\end{aligned}
$$

where

$$
H\left(r_{1}, r_{2}, q, \xi\right)=\frac{4 \pi^{2}}{q^{2}} \int_{0}^{\infty} \int_{0}^{\infty} G_{\xi}\left(x_{1}, x_{2}\right) J_{k-1}\left(\frac{4 \pi \sqrt{x_{1} r_{1}}}{q}\right) J_{k-1}\left(\frac{4 \pi \sqrt{x_{2} r_{2}}}{q}\right) d x_{1} d x_{2} .
$$

As above, we will take $\delta=Q^{-1}$, and later take $Q$ sufficiently large, hence it is sufficient to suppose that $\delta$ is smaller than $Z_{1} / m_{1} X_{1}$ and $Z_{2} / m_{2} Y_{2}$, so that $G_{\xi}\left(x_{1}, x_{2}\right)$ has essentially the same properties as $G\left(x_{1}, x_{2}\right)$. Integrating by parts 
many times and applying standard properties of the Bessel functions

$$
H\left(r_{1}, r_{2}, q, \xi\right) \ll \frac{X_{1} X_{2}}{q^{2}}\left(1+\frac{r_{1} X_{1}}{q^{2} Z_{1}^{2}}\right)^{-A}\left(1+\frac{r_{2} X_{2}}{q^{2} Z_{2}^{2}}\right)^{-B}
$$

for any $A, B>0$, and as a result we can restrict the summations to the ranges

$$
r_{1} \leq R_{1}:=Q^{2} Z_{1}^{2}\left(X_{1} X_{2}\right)^{\varepsilon} / X_{1}, r_{2} \leq R_{2}:=Q^{2} Z_{2}^{2}\left(X_{1} X_{2}\right)^{\varepsilon} / X_{2} .
$$

For such $r_{1}$ and $r_{2}$ we rewrite

$$
\begin{aligned}
H\left(r_{1}, r_{2}, q, \xi\right)=4 \pi^{2} q^{2} \int_{0}^{\infty} \int_{0}^{\infty} & G_{\xi}\left(q^{2} \xi_{1}, q^{2} \xi_{2}\right) \\
& \times J_{k-1}\left(4 \pi \sqrt{r_{1} \xi_{1}}\right) J_{k-1}\left(4 \pi \sqrt{r_{2} \xi_{2}}\right) d \xi_{1} d \xi_{2}
\end{aligned}
$$

to separate $q$ from $r_{1}$ and $r_{2}$, and hence

$$
\begin{aligned}
\mathscr{D}_{1}=\frac{4 \pi^{2}}{2 L} \int_{-\infty}^{\infty} \phi(\xi) \int_{0}^{\infty} \int_{0}^{\infty} & \sum_{q} q^{2} \omega(q) G_{\xi}\left(q^{2} \xi_{1}, q^{2} \xi_{2}\right) \\
\times & \sum_{r_{1}, r_{2}} a\left(r_{1}\right) J_{k-1}\left(4 \pi \sqrt{r_{1} \xi_{1}}\right) \overline{b\left(r_{2}\right)} J_{k-1}\left(4 \pi \sqrt{r_{1} \xi_{1}}\right) \\
\times & S\left(\bar{m}_{1} \bar{m}_{2}\left(m_{2} r_{1}-m_{1} r_{2}\right), h ; q\right) d \xi_{1} d \xi_{2} d \xi .
\end{aligned}
$$

Theorem 1.5 is now proved by using Fourier inversion to replace the Bessel functions by additive characters, at a cost of $Z_{1} Z_{2}$, and applying Theorem 1.6 to the average of the Kloosterman sums. We omit the details, which are similar to the proof of Theorem 1.4.

\section{Estimation of THE BILINEAR FORM}

All three parts of Theorem 1.2 are trivially true if $M \geq N^{1 / 2}$, so it will be sufficient to suppose that $M<N^{1 / 2}$. Where convenient we will use the notation $\sum^{\prime}$ to denote summation over squarefree values. We will first prove (1.10), so suppose for the present that the coefficients $\alpha_{m}$ are supported on squarefree integers. Applying Cauchy's inequality in $n$, we obtain

$$
\begin{aligned}
|\mathscr{B}(\alpha, \beta)|^{2} & \leq\|\beta\|^{2} \sum_{n \leq N}\left|\sum_{m \leq M} \alpha_{m} A(m n-1)\right|^{2} \\
& \ll\|\beta\|^{2} \sum_{n=1}^{\infty} e^{-4 \pi n / N}\left|\sum_{m \leq M} \alpha_{m} A(m n-1)\right|^{2} .
\end{aligned}
$$

Opening the square,

$$
|\mathscr{B}(\alpha, \beta)|^{2} \ll\|\beta\|^{2} \sum_{m_{1}, m_{2} \leq M} \alpha_{m_{1}} \bar{\alpha}_{m_{2}} \sum_{n=1}^{\infty} e^{-4 \pi n / N} A\left(m_{1} n-1\right) \overline{A\left(m_{2} n-1\right)}
$$

and the terms with $m_{1}=m_{2}$ contribute

$$
\|\beta\|^{2} \sum_{m \leq M}\left|\alpha_{m}\right|^{2} \sum_{n=1}^{\infty} e^{-4 \pi n / N}|A(m n-1)|^{2} \ll\|\alpha\|^{2}\|\beta\|^{2} N(M N)^{k-1+\varepsilon} .
$$


Denoting the remaining terms where $m_{1} \neq m_{2}$ by $\mathscr{D}$ we group the terms by the greatest common divisors $d=\left(m_{1}, m_{2}\right)$ and $D=\left(d^{\infty}, m_{1}-m_{2}\right)$ as in Theorem 1.4, writing $m_{1}=d m_{3}, m_{2}=d m_{4}$ as before. Thus

$$
\begin{aligned}
\mathscr{D}=\|\beta\|^{2} \sum_{d \leq M} \sum_{\substack{D\left|d^{\infty} \\
d\right| D}} \sum_{\substack{m_{3}, m_{4} \leq M / d \\
\left(m_{3}, m_{4}\right)=1, m_{3} \neq m_{4} \\
\left(m_{3}-m_{4}, d^{\infty}\right)=D / d}} \alpha_{d m_{3}} \bar{\alpha}_{d m_{4}} \\
\sum_{n=1}^{\infty} e^{-4 \pi n / N} A\left(d m_{3} n-1\right) \overline{A\left(d m_{4} n-1\right)} .
\end{aligned}
$$

This can be divided as $\mathscr{D}^{b}+\mathscr{D}^{\sharp}$ where $\mathscr{D}^{b}$ is the contribution from terms with $D>D_{0}$ and $\mathscr{D}^{\sharp}$ is the contribution from terms with $D \leq D_{0}$, where $D_{0} \in[1, M]$ will be chosen optimally later. For each $m_{3} \leq M / d$ there are $O(M / D)$ values $m_{4} \leq M / d$ such that $m_{4} \equiv m_{3}$ modulo $D / d$ but $m_{4} \neq m_{3}$, so for $\mathscr{D}^{b}$ we treat the sum over $n$ trivially and apply Cauchy's inequality in all other variables, obtaining

$$
\begin{aligned}
\mathscr{D}^{b} & \ll\|\beta\|^{2} M^{k-1+\varepsilon} N^{k+\varepsilon} \sum_{d \leq M}^{\prime} \sum_{\substack{D\left|d^{\infty} \\
d\right| D, D>D_{0}}} \sum_{\begin{array}{c}
m_{3}, m_{4} \leq M / d \\
\left(m_{3}, m_{4}\right)=1, m_{3} \neq m_{4} \\
\left(m_{3}-m_{4}, d^{\infty}\right)=D / d
\end{array}}^{\prime}\left|\alpha_{d m_{3}}\right|\left|\alpha_{d m_{4}}\right| \\
& \ll\|\alpha\|^{2}\|\beta\|^{2}(M N)^{k+\varepsilon}\left(\sum_{d \leq M}^{\prime} \frac{1}{d} \sum_{\substack{D\left|d^{\infty} \\
d\right| D, D>D_{0}}} \frac{1}{D}\right)^{1 / 2} .
\end{aligned}
$$

Factoring $D=d D_{1}$ we take $\varepsilon \in(0,1)$, and noting that $\left(1-x^{-\varepsilon}\right)^{-1} \ll x^{\varepsilon}$ for $x \geq 2$,

$$
\begin{aligned}
\sum_{d \leq M}^{\prime} \frac{1}{d} \sum_{\substack{D\left|d^{\infty} \\
d\right| D, D>D_{0}}} \frac{1}{D} & =\sum_{d \leq M}^{\prime} \frac{1}{d^{2}} \sum_{\substack{D_{1} \mid d^{\infty} \\
D_{1}>D_{0} / d}} \frac{1}{D_{1}} \\
& \leq D_{0}^{-1+\varepsilon} \sum_{d \leq M} \frac{1}{d^{1+\varepsilon}} \prod_{\substack{p \mid d \\
p \text { prime }}}\left(1-p^{-\varepsilon}\right)^{-1} \\
& \ll D_{0}^{-1+\varepsilon} \log M,
\end{aligned}
$$

so

$$
\mathscr{D}^{b} \ll\|\alpha\|^{2}\|\beta\|^{2}(M N)^{k+\varepsilon} D_{0}^{-1 / 2}
$$

For $\mathscr{D}^{\sharp}$ we apply Theorem 1.4 to the convolution over $n$ to bound

$$
\begin{aligned}
\mathscr{D}^{\sharp} \ll\|\beta\|^{2}(M N)^{k-1+\varepsilon} & \sum_{d \leq D_{0}} \sum_{\substack{D\left|d^{\infty} \\
d\right| D, D \leq D_{0}}} \sum_{\substack{m_{3}, m_{4} \leq M / d \\
\left(m_{3}, m_{4}\right)=1, m_{3} \neq m_{4} \\
\left(m_{3}-m_{4}, d^{\infty}\right)=D / d}}\left|\alpha_{d m_{3}}\right|\left|\alpha_{d m_{4}}\right| \\
& \times\left\{D\left(d m_{3} m_{4} N\right)^{1 / 2}+D^{1 / 4} d^{1 / 2}\left(m_{3} m_{4}\right)^{1 / 4} N^{3 / 4}\right\},
\end{aligned}
$$


so by Cauchy's inequality we obtain

$$
\begin{aligned}
\mathscr{D}^{\sharp} \ll & \|\alpha\|^{2}\|\beta\|^{2}(M N)^{k-1+\varepsilon} \\
& \times\left(M^{4} N \sum_{d \leq D_{0}}^{\prime} \frac{1}{d^{2}} \sum_{\substack{D\left|d^{\infty} \\
d\right| D, D \leq D_{0}}} D+M^{3} N^{3 / 2} \sum_{d \leq D_{0}}^{\prime} \frac{1}{d} \sum_{\substack{D\left|d^{\infty} \\
d\right| D, D \leq D_{0}}} D^{-1 / 2}\right)^{1 / 2} .
\end{aligned}
$$

For any $\varepsilon \in(0,1)$,

$$
\begin{aligned}
\sum_{d \leq D_{0}}^{\prime} \frac{1}{d^{2}} \sum_{\substack{D\left|d^{\infty} \\
d\right| D, D \leq D_{0}}} D & =\sum_{d \leq D_{0}}^{\prime} \frac{1}{d} \sum_{\substack{D_{1} \mid d^{\infty} \\
D_{1} \leq D_{0} / d}} D_{1} \\
& \leq D_{0}^{1+\varepsilon} \sum_{d \leq D_{0}} \frac{1}{d^{2+\varepsilon}} \prod_{p \mid d}\left(1-p^{-\varepsilon}\right)^{-1} \\
& \ll D_{0}^{1+\varepsilon}
\end{aligned}
$$

by similar arguments to the case above. We also have

$$
\begin{aligned}
\sum_{d \leq D_{0}}^{\prime} \frac{1}{d} \sum_{\substack{D\left|d^{\infty} \\
d\right| D, D \leq D_{0}}} D^{-1 / 2} & \leq \sum_{d \leq D_{0}}^{\prime} \frac{1}{d^{3 / 2}} \sum_{D_{1} \mid d^{\infty}} D_{1}^{-1 / 2} \\
& =\sum_{d \leq D_{0}}^{\prime} \frac{1}{d^{3 / 2}} \prod_{p \mid d}\left(1-p^{-1 / 2}\right)^{-1} \\
& \ll \log D_{0} .
\end{aligned}
$$

Collecting bounds,

$$
\mathscr{D}^{\sharp} \ll\|\alpha\|^{2}\|\beta\|^{2}\left\{D_{0}^{1 / 2} M^{2} N^{1 / 2}+M^{3 / 2} N^{3 / 4}\right\}(M N)^{k-1+\varepsilon} .
$$

Choosing $D_{0}=M^{-1} N^{1 / 2}$ we obtain (1.10) from (6.1), (6.2) and (6.3).

To remove the restriction to squarefree $m$ and prove (1.9), factor each $m$ uniquely as $l^{2} m^{\prime}$ where $m^{\prime}$ is squarefree. Then

$$
\mathscr{B}(\alpha, \beta)=\sum_{l \leq M^{1 / 2}} \sum_{n \leq N} \beta_{n} \sum_{m^{\prime} \leq M l^{-2}}^{\prime} \alpha_{l^{2} m^{\prime}} A\left(m^{\prime} l^{2} n-1\right) .
$$

Dividing the sum over $l$ at some $L$ to be chosen later, the contribution from $l>L$ can be bounded as

$$
\begin{aligned}
& \sum_{L<l \leq M^{1 / 2}} \sum_{n \leq N} \beta_{n} \sum_{m^{\prime} \leq M l^{-2}}^{\prime} \alpha_{l^{2} m^{\prime}} A\left(m^{\prime} l^{2} n-1\right) \\
& \left.\ll \sum_{L<l \leq M^{1 / 2}} \sum_{n \leq N}\left|\beta_{n}\right|^{2} \sum_{m^{\prime} \leq M l^{-2}}^{\prime}\left|\alpha_{l^{2} m^{\prime}}\right|^{2}\right\}^{1 / 2} \\
& \quad \times\left\{\sum_{L<l \leq(2 M)^{1 / 2}} \sum_{n \leq N} \sum_{m^{\prime} \leq M l^{-2}}^{\prime} 1\right\}^{1 / 2}(M N)^{(k-1) / 2+\varepsilon} \\
& \ll\|\alpha\|\|\beta\|(M N)^{k / 2+\varepsilon} L^{-1 / 2} .
\end{aligned}
$$


The contribution from small $l$ is

$\sum_{l \leq L} \sum_{n \leq N} \beta_{n} \sum_{m^{\prime} \leq M l^{-2}}^{\prime} \alpha_{l^{2} m^{\prime}} A\left(m^{\prime} l^{2} n-1\right)=\sum_{l \leq L} \sum_{n \leq N l^{2}} \beta_{n l^{-2}} \sum_{m^{\prime} \leq M l^{-2}}^{\prime} \alpha_{l^{2} m^{\prime}} A\left(m^{\prime} n-1\right)$

and we view the inner two sums as a bilinear form, where $\beta$ is understood to be zero unless $l^{2} \mid n$. Since $m^{\prime}$ varies over squarefree values we can apply (1.10), which gives

$$
\begin{aligned}
\sum_{l \leq L} \sum_{n \leq N} \beta_{n} \sum_{m^{\prime} \leq M l^{-2}}^{\prime} \alpha_{l^{2} m^{\prime}} A\left(m^{\prime} l^{2} n-1\right) & \\
\ll & \sum_{l \leq L}\left\{\sum_{n \leq N}\left|\beta_{n}\right|^{2}\right\}^{1 / 2}\left\{\sum_{m^{\prime} \leq M l^{-2}}^{\prime}\left|\alpha_{l^{2} m^{\prime}}\right|^{2}\right\}^{1 / 2} \\
& \times\left\{N^{1 / 2} l+\left(M / l^{2}\right)^{3 / 4}\left(N l^{2}\right)^{3 / 8}\right\}(M N)^{(k-1) / 2+\varepsilon} \\
\ll & \|\alpha\|\|\beta\|\left\{N^{1 / 2} L^{3 / 2}+M^{3 / 4} N^{3 / 8}\right\}(M N)^{(k-1) / 2+\varepsilon} .
\end{aligned}
$$

Balancing (6.4) and (6.5) by choosing $L=M^{1 / 4}$ we obtain (1.9).

To prove (1.11) we note that with the additional hypotheses that $\alpha_{m} \ll m^{\varepsilon}$ the bound (6.4) can be improved to give

$$
\begin{aligned}
& \sum_{L<l \leq M^{1 / 2}} \sum_{n \leq N} \beta_{n} \sum_{m^{\prime} \leq M l^{-2}}^{\prime} \alpha_{l^{2} m^{\prime}} A\left(m^{\prime} l^{2} n-1\right) \\
\ll & \sum_{L<l \leq M^{1 / 2}} \sum_{n \leq N}\left|\beta_{n}\right| M l^{-2}(M N)^{(k-1) / 2+\varepsilon} \\
\ll & \|\beta\|(M N)^{k / 2+\varepsilon} M^{1 / 2} L^{-1}
\end{aligned}
$$

and (6.5) can be improved to give

$$
\begin{aligned}
& \sum_{l \leq L} \sum_{n \leq N} \beta_{n} \sum_{m^{\prime} \leq M l^{-2}}^{\prime} \alpha_{l^{2} m^{\prime}} A\left(m^{\prime} l^{2} n-1\right) \\
\ll & \|\beta\|\left\{(M N)^{1 / 2} L+M^{5 / 4} N^{3 / 8}\right\}(M N)^{(k-1) / 2+\varepsilon}
\end{aligned}
$$

so that optimising at $L=M^{1 / 4}$ as before we obtain (1.11).

\section{Proof of Corollary 1.3}

First note that

$$
\begin{aligned}
\mathscr{B}_{g}(\alpha, \beta) & =\sum_{M_{1}<m \leq M_{2}} \sum_{n} \alpha_{m} \beta_{n} \frac{g(m n)}{(m n)^{(k-1) / 2}} A(m n-1)\left(1+O\left((m n)^{-1}\right)\right) \\
& =\sum_{M_{1}<m \leq M_{2}} \sum_{n} \alpha_{m} \beta_{n} \frac{g(m n)}{(m n)^{(k-1) / 2}} A(m n-1)+O\left(X^{\varepsilon}\right) .
\end{aligned}
$$

In the remaining expression the sum over $n$ can be broken into $O(\log X)$ ranges $N<n \leq 2 N$ for $N$ ranging between $X / 2 M_{2}$ and $4 X / M_{1}$, and for each such interval the function $g(m n)$ restricts the sum over $m$ to $M<m \leq 16 M$, where $M=X / 4 N$. In this way it is sufficient to consider $O(\log X)$ sums of the form

$$
\mathscr{B}_{g, M, N}(\alpha, \beta)=\sum_{M<m \leq 16 M} \sum_{N<n \leq 2 N} \alpha_{m} \beta_{n} g(m n)(m n)^{-(k-1) / 2} A(m n-1) .
$$


Defining the Mellin transform

$$
\mathscr{M}(s)=\int_{0}^{\infty} g(x) x^{-(k-1) / 2} x^{s-1} d x
$$

the compact support of $g$ implies that $\mathscr{M}(s)$ is an entire function; furthermore, we can integrate by parts $\nu$ times, to obtain

$$
\mathscr{M}(s)=\frac{(-1)^{\nu}}{\prod_{j=0}^{\nu-1}(s-(k-1) / 2+j)} \int_{0}^{\infty} g^{(\nu)}(x) x^{s-(k-1) / 2-1+\nu} d x
$$

for any $\nu>0$, as long as the denominator is non-zero. It follows that for $s=\sigma+i t$ and $t \neq 0$,

$$
\mathscr{M}(s) \ll X^{\sigma-(k-1) / 2} \min \left\{1,|t|^{-\nu}\left(\frac{X}{Y}\right)^{\nu-1}\right\}
$$

for any $\nu>0$, where the first bound follows by estimating without integrating by parts, and the second uses the fact that $g^{(\nu)}(x)$ vanishes outside two intervals of length $Y$ if $\nu>0$. The transform can now be inverted to give

$$
g(y) y^{-(k-1) / 2}=\frac{1}{2 \pi i} \int_{\sigma-i \infty}^{\sigma+i \infty} \mathscr{M}(s) y^{-s} d s
$$

where we are free to choose $\sigma=0$, and

$$
\mathscr{B}_{g, M, N}(\alpha, \beta)=\frac{1}{2 \pi} \int_{-\infty}^{\infty} \mathscr{M}(i t) \sum_{M<m \leq 16 M} \sum_{N<n \leq 2 N} \alpha_{m} m^{-i t} \beta_{n} n^{-i t} A(m n-1) d t .
$$

Applying (1.11) we obtain

$$
\mathscr{B}_{g, M, N}(\alpha, \beta) \ll\left\{X M^{-1 / 4}+X^{7 / 8} M^{3 / 8}\right\} X^{(k-1) / 2+\varepsilon} \int_{-\infty}^{\infty}|\mathscr{M}(i t)| d t .
$$

The integral can be broken into three parts; we apply (7.2) with $\nu=0$ for $|t|<1$, with $\nu=1$ for $1<|t|<X / Y$ and for any $\nu \geq 2$ for $|t|>X / Y$ to obtain

$$
\begin{aligned}
\int_{-\infty}^{\infty}|\mathscr{M}(i t)| d t & \ll X^{-(k-1) / 2}\left\{\int_{|t|<1} d t+\int_{1<|t|<X / Y} \frac{d t}{|t|}+\left(\frac{X}{Y}\right)^{\nu-1} \int_{|t|>X / Y} \frac{d t}{|t|^{\nu}}\right\} \\
& \ll X^{-(k-1) / 2+\varepsilon} .
\end{aligned}
$$

Corollary 1.3 now follows from (7.3) by recalling that the values of $M$ to be considered are no smaller than $M_{1} / 16$ nor larger than $M_{2}$. 


\section{An estimate for a sum of Fourier COefficients}

The summation formula (5.7) is sufficient to prove the following auxiliary lemma.

Lemma 8.1. Let $g(x)$ be as in the statement of Corollary 1.3 and let $\phi(x)$ be any smooth function such that $\phi^{(\nu)}(x) \ll x^{-\nu}$ for $\nu=0,1,2, \ldots$ Then for any $\varepsilon>0$,

$$
\sum_{n} g(m n) \phi(n) a(m n-1) \ll m^{1 / 2}(X / Y)^{3 / 2}(m X)^{\varepsilon} .
$$

The essential point here is that as long as $m<X^{2 / 3-\eta}$ for some $\eta>0$ this bound is non-trivial for some $Y$ sufficiently close to $X$, which is sufficient to eliminate the cases $k=1$ and $k=2$ mentioned in the introduction.

Proof. Using additive characters and applying (5.7), the sum is

$$
\begin{aligned}
& \frac{1}{m} \sum_{q \mid m} \sum_{u(\bmod q)}^{*} e_{q}(u) \sum_{r} G(r) a(r) e_{q}(u r) \\
& \quad=\frac{2 \pi i^{k}}{m} \sum_{q \mid m} \frac{1}{q} \sum_{u(\bmod q)}^{*} e_{q}(u) \sum_{h} a(h) e_{q}(-\bar{u} h) \int_{0}^{\infty} G(x) J_{k-1}\left(\frac{4 \pi \sqrt{x h}}{q}\right) d x
\end{aligned}
$$

where $G(r)=g(r+1) \phi((r+1) / m)$. Integrating by parts and using the recurrence relation for the Bessel function $J_{k-1}$ the Hankel transform can be bounded by

$$
\int_{0}^{\infty} G(x) J_{k-1}(\sqrt{y x}) d x \ll X\left(1+\frac{y Y^{2}}{X}\right)^{-A}
$$

for any $A>0$, so the summation in $h$ can be truncated at $h<q^{2} X^{1+\varepsilon} Y^{-2}$ at a cost of an error term $O\left(q^{2} X^{-100}\right)$, say. In the remaining terms we use the standard bound $J_{\nu}(x) \ll x^{-1 / 2}$ to bound the transform as $O\left(q^{1 / 2} h^{-1 / 4} X^{3 / 4}\right)$, and

$$
\sum_{n} g(m n) \phi(n) a(m n-1) \ll X^{3 / 4} \sum_{q \mid m} \frac{1}{m q^{1 / 2}} \sum_{h \leq q^{2} X^{1+\varepsilon} Y^{-2}} \frac{|a(h)|}{h^{1 / 4}}\left|\sum_{u(\bmod q)}^{*} e_{q}(u-\bar{u} h)\right| .
$$

The lemma is now proved by using Weil's bound for the Kloosterman sum.

\section{Proof of Theorem 1.1.}

The proof of Theorem 1.1 requires a version of Vaughan's identity, which uses the following convenient notation. Let $\phi$ be a $\mathscr{C}^{\infty}$ test function which is compactly supported on $[-2,2]$, is identically one on $[-1 / 2,1 / 2]$, and is such that $\phi(x)+$ $\phi\left(x^{-1}\right)=1$. We introduce the notations

$$
\phi^{A}(x)=\phi\left(\frac{x}{A}\right), \phi_{A}(x)=\phi\left(\frac{A}{x}\right), \phi_{A}^{B}(x)=\phi\left(\frac{x}{B}\right) \phi\left(\frac{A}{x}\right)
$$

for any $A, B>0$. Loosely speaking, the first one smoothly restricts $x$ to values less than $A$, the second to $x$ greater than $A$, and the third to $x$ larger than $A$ but less than $B$. Note that $\phi^{A}(x)+\phi_{A}(x)=1$ for all $x$. 
Lemma 9.1. If $n>\max \{K, 2 M\}$, then

$$
\Lambda(n)=\sum_{\substack{k \mid n \\ k \leq K}} \mu(k) \log \left(\frac{n}{k}\right)-\sum_{\substack{k m \mid n \\ k \leq K}} \mu(k) \Lambda(m) \phi^{M}(m)+\sum_{\substack{k m \mid n \\ k>K}} \mu(k) \Lambda(m) \phi_{M}(m)
$$

and

$$
\mu(n)=-\sum_{\substack{k m \mid n \\ k \leq K}} \mu(k) \mu(m) \phi^{M}(m)+\sum_{\substack{k m \mid n \\ k>K}} \mu(k) \mu(m) \phi_{M}(m) .
$$

Proof. This is almost precisely the same as in [13]. To prove (9.1) we expand $\Lambda$ as

$$
\Lambda(n)=\sum_{k \mid n} \mu(k) \log \left(\frac{n}{k}\right)=\sum_{\substack{k \mid n \\ k \leq K}} \mu(k) \log \left(\frac{n}{k}\right)+\sum_{\substack{k \mid n \\ k>K}} \mu(k) \log \left(\frac{n}{k}\right) .
$$

The second term is

$$
\begin{aligned}
& \sum_{\substack{k m \mid n \\
k>K}} \mu(k) \Lambda(m) \phi^{M}(m)+\sum_{\substack{k m \mid n \\
k>K}} \mu(k) \Lambda(m) \phi_{M}(m) \\
= & \sum_{m \mid n} \Lambda(m) \phi^{M}(m) \sum_{k \mid n / m} \mu(k)-\sum_{\substack{k m \mid n \\
k \leq K}} \mu(k) \Lambda(m) \phi^{M}(m)+\sum_{\substack{k m \mid n \\
k>K}} \mu(k) \Lambda(m) \phi_{M}(m) .
\end{aligned}
$$

The inner sum in the first term vanishes unless $n=m$. This cannot happen if $n>2 M$, which proves (9.1). The proof of (9.2) uses the expansion

$$
\mu(n)=\sum_{k m \mid n} \mu(k) \mu(m)=\sum_{\substack{k m \mid n \\ k \leq K}} \mu(k) \mu(m)+\sum_{\substack{k m \mid n \\ k>K}} \mu(k) \mu(m)
$$

and continues in a similar manner to the argument for (9.1).

Consider $\mathscr{T}_{f}(X)$ as in (1.5), for $X>2$, and the related sums

$$
\mathscr{T}_{f}^{*}(X)=\sum_{X<n \leq 2 X} \Lambda(n) a(n-1), \mathscr{T}_{g}=\sum_{n} g(n) \Lambda(n) a(n-1)
$$

where $g(x)$ obeys the hypotheses of Corollary 1.3 for some $Y$ between 1 and $X / 2$ to be chosen optimally later. Recall that this means $g$ is $\mathscr{C}^{\infty}$ on $(0, \infty)$, identically one on $[X, 2 X]$, supported on $[X-Y, 2 X+Y]$, and with derivatives of all orders bounded by

$$
g^{(\nu)}(x) \ll Y^{-\nu}, \nu=0,1,2 \ldots
$$

Then

$$
\mathscr{T}_{f}^{*}(X)=\mathscr{T}_{g}+O\left(Y X^{\varepsilon}\right) .
$$

Applying (9.1) to $\mathscr{T}_{g}$ we obtain

$$
\mathscr{T}_{g}=\mathscr{S}_{1}-\mathscr{T}_{1}+\mathscr{U}_{1}
$$


where

$$
\begin{aligned}
& \mathscr{S}_{1}=\sum_{k_{1} \leq K_{1}} \sum_{n_{1}} g\left(k_{1} n_{1}\right) \mu\left(k_{1}\right) \log n_{1} a\left(k_{1} n_{1}-1\right), \\
& \mathscr{T}_{1}=\sum_{k_{1} \leq K_{1}} \sum_{m_{1}, n_{1}} g\left(k_{1} m_{1} n_{1}\right) \mu\left(k_{1}\right) \Lambda\left(m_{1}\right) \phi^{M_{1}}\left(m_{1}\right) a\left(k_{1} m_{1} n_{1}-1\right), \\
& \mathscr{U}_{1}=\sum_{k_{1}>K_{1}} \sum_{m_{1}, n_{1}} g\left(k_{1} m_{1} n_{1}\right) \mu\left(k_{1}\right) \Lambda\left(m_{1}\right) \phi_{M_{1}}\left(m_{1}\right) a\left(k_{1} m_{1} n_{1}-1\right)
\end{aligned}
$$

for $K_{1}$ and $M_{1}$ to be chosen later such that $K_{1}<X / 2$ and $M_{1}<X / 4$, so that the hypotheses of Lemma 9.1 are satisfied for all $n$ under consideration.

The first sum $\mathscr{S}_{1}$ is smooth in $n_{1}$, so applying Lemma 8.1 gives us

$$
\mathscr{S}_{1} \ll\left(K_{1} X / Y\right)^{3 / 2} X^{\varepsilon} .
$$

By defining $m=k_{1}$ and $n=m_{1} n_{1}$, and

$$
\alpha_{m}=\mu(m), \beta_{n}=\sum_{m_{1} n_{1}=n} \Lambda\left(m_{1}\right) \phi_{M_{1}}\left(m_{1}\right),
$$

we can write $\mathscr{U}_{1}$ in the form (1.12), and by Corollary 1.3 we obtain

$$
\mathscr{U}_{1}=\sum_{K_{1}<m \leq 6 X M_{1}^{-1}} \sum_{n} \alpha_{m} \beta_{n} g(m n) a(m n-1) \ll\left\{X K_{1}^{-1 / 4}+X^{7 / 8}\left(X M_{1}^{-1}\right)^{3 / 8}\right\} X^{\varepsilon} .
$$

For $\mathscr{T}_{1}$ we introduce a new parameter $M_{2}$ to be chosen later such that $M_{2}<M_{1} / 16$, so $\phi^{M_{1}}\left(m_{1}\right)=1$ whenever $\phi^{4 M_{2}}\left(m_{1}\right)$ is non-zero. Separating $\mathscr{T}_{1}$ into two parts according to the size of $m_{1}$ gives

$$
\begin{aligned}
\mathscr{T}_{1}= & \sum_{k_{1} \leq K_{1}} \sum_{m_{1}, n_{1}} g\left(k_{1} m_{1} n_{1}\right) \mu\left(k_{1}\right) \Lambda\left(m_{1}\right) \phi_{4 M_{2}}^{M_{1}}\left(m_{1}\right) a\left(k_{1} m_{1} n_{1}-1\right) \\
& +\sum_{k_{1} \leq K_{1}} \sum_{m_{1}, n_{1}} g\left(k_{1} m_{1} n_{1}\right) \mu\left(k_{1}\right) \Lambda\left(m_{1}\right) \phi^{4 M_{2}}\left(m_{1}\right) a\left(k_{1} m_{1} n_{1}-1\right) .
\end{aligned}
$$

In the second term the sum in $n_{1}$ is smooth, and $k_{1} m_{1} \leq 8 K_{1} M_{2}$, so applying Lemma 8.1 to the sum in $n_{1}$ gives us

$$
\begin{aligned}
\mathscr{T}_{1}=\sum_{k_{1} \leq K_{1}} \sum_{m_{1}, n_{1}} g\left(k_{1} m_{1} n_{1}\right) \mu\left(k_{1}\right) \Lambda\left(m_{1}\right) \phi_{4 M_{2}}^{M_{1}}\left(m_{1}\right) & a\left(k_{1} m_{1} n_{1}-1\right) \\
& +O\left(\left(K_{1} M_{2} X / Y\right)^{3 / 2} X^{\varepsilon}\right) .
\end{aligned}
$$

We suppose that $K_{1} M_{2} \leq X^{1 / 2}$, which will be justified later, so this error is $O\left(X^{9 / 4+\varepsilon} Y^{-3 / 2}\right)$.

We next apply (9.1) again to the variable $m_{1}$ in the remaining sum; since $m_{1}$ is in the range $2 M_{2}<m_{1}<2 M_{1}$ by the support of $\phi_{4 M_{2}}^{M_{1}}$ the hypotheses of Lemma 9.1 are satisfied if we later choose $K_{2}<2 M_{2}$. Thus

$$
\begin{aligned}
\Lambda\left(m_{1}\right)=\sum_{\substack{k_{2} \mid m_{1} \\
k_{2} \leq K_{2}}} \mu\left(k_{2}\right) \log \left(\frac{m_{1}}{k_{2}}\right) & -\sum_{\substack{k_{2} m_{2} \mid m_{1} \\
k_{2} \leq K_{2}}} \mu\left(k_{2}\right) \Lambda\left(m_{2}\right) \phi^{M_{2}}\left(m_{2}\right) \\
& +\sum_{\substack{k_{2} m_{2} \mid m_{1} \\
k_{2}>K_{2}}} \mu\left(k_{2}\right) \Lambda\left(m_{2}\right) \phi_{M_{2}}\left(m_{2}\right)
\end{aligned}
$$


and

$$
\mathscr{T}_{1}=\mathscr{S}_{2}-\mathscr{T}_{2}+\mathscr{U}_{2}+O\left(Y^{-3 / 2} X^{9 / 4+\varepsilon}\right)
$$

where

$$
\begin{aligned}
& \mathscr{S}_{2}=\sum_{\substack{k_{1} \leq K_{1} \\
k_{2} \leq K_{2}}} \mu\left(k_{1}\right) \mu\left(k_{2}\right) \sum_{n_{1}, n_{2}} g\left(k_{1} k_{2} n_{1} n_{2}\right) \phi_{4 M_{2}}^{M_{1}}\left(k_{2} n_{2}\right) \log n_{2} a\left(k_{1} k_{2} n_{1} n_{2}-1\right), \\
& \mathscr{T}_{2}=\sum_{\substack{k_{1} \leq K_{1} \\
k_{2} \leq K_{2}}} \mu\left(k_{1}\right) \mu\left(k_{2}\right) \sum_{m_{2}, n_{1}, n_{2}} g\left(k_{1} k_{2} m_{2} n_{1} n_{2}\right) \Lambda\left(m_{2}\right) \\
& \times \phi_{4 M_{2}}^{M_{1}}\left(k_{2} m_{2} n_{2}\right) \phi^{M_{2}}\left(m_{2}\right) a\left(k_{1} k_{2} m_{2} n_{1} n_{2}-1\right), \\
& \mathscr{U}_{2}=\sum_{\substack{k_{1} \leq K_{1} \\
k_{2}>K_{2}}} \mu\left(k_{1}\right) \mu\left(k_{2}\right) \sum_{m_{2}, n_{1}, n_{2}} g\left(k_{1} k_{2} m_{2} n_{1} n_{2}\right) \Lambda\left(m_{2}\right) \\
& \times \phi_{4 M_{2}}^{M_{1}}\left(k_{2} m_{2} n_{2}\right) \phi_{M_{2}}\left(m_{2}\right) a\left(k_{1} k_{2} m_{2} n_{1} n_{2}-1\right) .
\end{aligned}
$$

We treat the estimation of $\mathscr{S}_{2}$ in a similar manner to $\mathscr{S}_{1}$, although the details are slightly more complicated. Let $\sum f_{j}(x)=1$ be a smooth partition of unity on $(0, \infty)$ where the functions $f_{j}(x)$ are supported on intervals $\left[N_{j} / 2,2 N_{j}\right]$, for $N_{j}=2^{j}$, and where

$$
f_{j}^{(\nu)}(x) \ll x^{-\nu}, \nu=0,1,2, \ldots
$$

Using this partition of unity in both $n_{1}$ and $n_{2}, \mathscr{S}_{2}$ can be written as $O\left(\log ^{2} X\right)$ sums of the form

$$
\sum_{\substack{k_{1} \leq K_{1} \\ k_{2} \leq K_{2}}} \mu\left(k_{1}\right) \mu\left(k_{2}\right) \sum_{n_{1}, n_{2}} f_{j_{1}}\left(n_{1}\right) f_{j_{2}}\left(n_{2}\right) g\left(k_{1} k_{2} n_{1} n_{2}\right) \phi_{4 M_{2}}^{M_{1}}\left(k_{2} n_{2}\right) \log n_{2} a\left(k_{1} k_{2} n_{1} n_{2}-1\right)
$$

where $X / 2 k_{1} k_{2}<N_{j_{1}} N_{j_{2}}<3 X / k_{1} k_{2}$ from the support of $g$. It follows that at least one of $N_{j_{1}}$ and $N_{j_{2}}$ is larger than $\left(X / 2 k_{1} k_{2}\right)^{1 / 2}$. If $N_{j_{1}}$ is larger than this, then we apply Lemma 8.1 with $n=n_{1}$ and $m=k_{1} k_{2} n_{2}$; if $N_{j_{2}}$ is larger, then the roles of $n_{1}$ and $n_{2}$ are switched. The values of $m$ range up to $\left(X K_{1} K_{2}\right)^{1 / 2}$, and

$$
\mathscr{S}_{2} \ll\left(\left(X K_{1} K_{2}\right)^{1 / 2} X / Y\right)^{3 / 2} X^{\varepsilon} \ll\left(K_{1} K_{2}\right)^{3 / 4} Y^{-3 / 2} X^{9 / 4+\varepsilon} .
$$

The estimation of $\mathscr{U}_{2}$ is also similar to that of $\mathscr{U}_{1}$; however, we must remove the extra function $\phi_{4 M_{1}}^{M_{2}}\left(k_{2} m_{2} n_{2}\right)$ by Mellin inversion. For $B \geq A / 4$ we define

$$
\mathscr{M}_{A}^{B}(s)=\int_{0}^{\infty} \phi_{A}^{B}(x) x^{s-1} d x
$$

which is bounded by $O\left(\left(A^{\sigma}+B^{\sigma}\right) \log 4 B / A\right)$; we can integrate by parts $\nu$ times as in (7.2) to give another bound:

$$
\begin{aligned}
\mathscr{M}_{A}^{B}(s) & =\frac{(-1)^{\nu}}{s(s+1) \cdots(s-1+\nu)} \int_{0}^{\infty} \frac{d^{\nu}}{d x^{\nu}}\left\{\phi\left(\frac{x}{B}\right) \phi\left(\frac{A}{x}\right)\right\} x^{s-1+\nu} d x \\
& \ll\left\{A^{\sigma}+B^{\sigma}\right\}|t|^{-\nu},
\end{aligned}
$$


since the $\nu$-th derivative vanishes outside the intervals $[A / 2,2 A]$ and $[B / 2,2 B]$. The two bounds combine to give

$$
\mathscr{M}_{A}^{B}(s) \ll\left\{A^{\sigma}+B^{\sigma}\right\} \min \left\{\log \frac{4 B}{A},|t|^{-\nu}\right\} .
$$

In the present case $A=4 M_{2}$ and $B=M_{1}$; the variable $k_{2}$ is between $K_{2}$ and $4 M_{1} M_{2}^{-1}$ by the support of $\phi_{4 M_{1}}^{M_{2}}$, so

$$
\begin{array}{r}
\mathscr{U}_{2}=\frac{1}{2 \pi i} \int_{-i \infty}^{i \infty} \mathscr{M}_{4 M_{2}}^{M_{1}}(s) \sum_{\substack{k_{1} \leq K_{1} \\
K_{2}<k_{2} \leq 4 M_{1} M_{2}^{-1}}} \mu\left(k_{1}\right) \mu\left(k_{2}\right) k_{2}^{-s} \sum_{m_{2}, n_{1}, n_{2}} g\left(k_{1} k_{2} m_{2} n_{1} n_{2}\right) \Lambda\left(m_{2}\right) \\
\times\left(m_{2} n_{2}\right)^{-s} \phi_{M_{2}}\left(m_{2}\right) a\left(k_{1} k_{2} m_{2} n_{1} n_{2}-1\right) d s .
\end{array}
$$

Defining $m=k_{2}, n=k_{1} m_{2} n_{1} n_{2}$ and

$$
\alpha_{m}(t)=\mu(m) m^{-i t}, \beta_{n}(t)=\sum_{\substack{k_{1} m_{2} n_{1} n_{2}=n \\ k_{1} \leq K}} \mu\left(k_{1}\right) \Lambda\left(m_{2}\right) \phi_{M_{2}}\left(m_{2}\right)\left(m_{2} n_{2}\right)^{-i t}
$$

this is

$$
\mathscr{U}_{2}=\frac{1}{2 \pi} \int_{-\infty}^{\infty} \mathscr{M}_{4 M_{2}}^{M_{1}}(i t) \sum_{k_{2}<m \leq M_{1} M_{2}^{-1}} \sum_{n} \alpha_{m}(t) \beta_{n}(t) g(m n) a(m n-1) d t,
$$

so Corollary 1.3 and (9.8) now give

$$
\begin{aligned}
\mathscr{U}_{2} & \ll\left\{X K_{2}^{-1 / 4}+X^{7 / 8}\left(M_{1} M_{2}^{-1}\right)^{3 / 8}\right\} X^{\varepsilon} \int_{-\infty}^{\infty}\left|\mathscr{M}_{4 M_{2}}^{M_{1}}(i t)\right| d t \\
& \ll\left\{X K_{2}^{-1 / 4}+X^{7 / 8}\left(M_{1} M_{2}^{-1}\right)^{3 / 8}\right\} X^{\varepsilon} .
\end{aligned}
$$

For $\mathscr{T}_{2}$ we again separate into large and small parts, using $M_{3}<M_{2} / 16$ to be chosen later, so

$$
\begin{aligned}
\mathscr{T}_{2}= & \sum_{\substack{k_{1} \leq K_{1} \\
k_{2} \leq K_{2}}} \mu\left(k_{1}\right) \mu\left(k_{2}\right) \sum_{m_{2}, n_{1}, n_{2}} g\left(k_{1} k_{2} m_{2} n_{1} n_{2}\right) \Lambda\left(m_{2}\right) \\
& \times \phi_{4 M_{2}}^{M_{1}}\left(k_{2} m_{2} n_{2}\right) \phi_{4 M_{3}}^{M_{2}}\left(m_{2}\right) a\left(k_{1} k_{2} m_{2} n_{1} n_{2}-1\right) \\
& +\sum_{\substack{k_{1} \leq K_{1} \\
k_{2} \leq K_{2}}} \mu\left(k_{1}\right) \mu\left(k_{2}\right) \sum_{m_{2}, n_{1}, n_{2}} g\left(k_{1} k_{2} m_{2} n_{1} n_{2}\right) \Lambda\left(m_{2}\right) \\
& \times \phi_{4 M_{2}}^{M_{1}}\left(k_{2} m_{2} n_{2}\right) \phi^{4 M_{3}}\left(m_{2}\right) a\left(k_{1} k_{2} m_{2} n_{1} n_{2}-1\right) .
\end{aligned}
$$

The second term here is smooth in $n_{1}$ and $n_{2}$, and $k_{1} k_{2} m_{2} \leq 8 K_{1} K_{2} M_{3}$, so at least one of $n_{1}$ and $n_{2}$ is larger than $\left(X / K_{1} K_{2} M_{3}\right)^{1 / 2}$. We restrict to $K_{1}, K_{2}, M_{3}$ such that $K_{1} K_{2} M_{3} \leq X^{1 / 3}$, and using the partition of unity $\sum_{j} f_{j}(x)=1$ as in the proof of (9.7) we obtain

$$
\begin{aligned}
\mathscr{T}_{2}=\sum_{\substack{k_{1} \leq K_{1} \\
k_{2} \leq K_{2}}} \mu\left(k_{1}\right) \mu\left(k_{2}\right) \sum_{m_{2}, n_{1}, n_{2}} g\left(k_{1} k_{2} m_{2} n_{1} n_{2}\right) \Lambda\left(m_{2}\right) \\
\times \phi_{4 M_{2}}^{M_{1}}\left(k_{2} m_{2} n_{2}\right) \phi_{4 M_{3}}^{M_{2}}\left(m_{2}\right) a\left(k_{1} k_{2} m_{2} n_{1} n_{2}-1\right) \\
+O\left(\left(\left(X K_{1} K_{2} M_{3}\right)^{1 / 2} X / Y\right)^{3 / 2} X^{\varepsilon}\right)
\end{aligned}
$$


by applying Lemma 8.1 . Note that (9.7) can be absorbed into this error term, as can the error in (9.6).

We now apply Lemma 9.1 a third time, to expand $\Lambda\left(m_{2}\right)$, using $M_{3}$ as defined above and $K_{3}$ to be chosen later subject to the condition that $K_{3} \leq 2 M_{3}$. Thus, from the support of $\phi_{4 M_{3}}^{M_{2}}$ we have $2 M_{3}<m_{2} \leq 2 M_{2}$, so the hypotheses of Lemma 9.1 are satisfied. This gives

$$
\mathscr{T}_{2}=\mathscr{S}_{3}-\mathscr{T}_{3}+\mathscr{U}_{3}+O\left(\left(K_{1} K_{2} M_{3}\right)^{3 / 4} Y^{-3 / 2} X^{9 / 4+\varepsilon}\right)
$$

where

$$
\begin{aligned}
& \mathscr{S}_{3}=\sum_{\substack{k_{1} \leq K_{1} \\
k_{2} \leq K_{2} \\
k_{3} \leq K_{3}}} \mu\left(k_{1}\right) \mu\left(k_{2}\right) \mu\left(k_{3}\right) \sum_{n_{1}, n_{2}, n_{3}} g\left(k_{1} k_{2} k_{3} n_{1} n_{2} n_{3}\right) \\
& \quad \times \phi_{4 M_{2}}^{M_{1}}\left(k_{2} k_{3} n_{2} n_{3}\right) \phi_{4 M_{3}}^{M_{2}}\left(k_{3} n_{3}\right) \log n_{3} a\left(k_{1} k_{2} k_{3} n_{1} n_{2} n_{3}-1\right), \\
& \mathscr{T}_{3}=\sum_{\substack{k_{1} \leq K_{1} \\
k_{2} \leq K_{2} \\
k_{3} \leq K_{3}}} \mu\left(k_{1}\right) \mu\left(k_{2}\right) \mu\left(k_{3}\right) \sum_{m_{3}} \Lambda\left(m_{3}\right) \phi^{M_{3}}\left(m_{3}\right) \sum_{n_{1}, n_{2}, n_{3}} g\left(k_{1} k_{2} k_{3} m_{3} n_{1} n_{2} n_{3}\right) \\
& \qquad \phi_{4 M_{2}}^{M_{1}}\left(k_{2} k_{3} m_{3} n_{2} n_{3}\right) \phi_{4 M_{3}}^{M_{2}}\left(k_{3} m_{3} n_{3}\right) a\left(k_{1} k_{2} k_{3} m_{3} n_{1} n_{2} n_{3}-1\right), \\
& \mathscr{U}_{3}=\sum_{\substack{k_{1} \leq K_{1} \\
k_{2} \leq K_{2} \\
k_{3}>K_{3}}} \mu\left(k_{1}\right) \mu\left(k_{2}\right) \mu\left(k_{3}\right) \sum_{m_{3}} \Lambda\left(m_{3}\right) \phi_{M_{3}}\left(m_{3}\right) \sum_{n_{1}, n_{2}, n_{3}} g\left(k_{1} k_{2} k_{3} m_{3} n_{1} n_{2} n_{3}\right)
\end{aligned}
$$

In the third expression we have $k_{3}>K_{3}$ but also $k_{3} m_{3} n_{3} \leq 2 M_{2}$ and $m_{3}>M_{3} / 2$ from the supports of the functions $\phi$, so $k_{3} \leq 4 M_{2} M_{3}^{-1}$. Thus as above we can use Mellin inversion to separate the variables in $\phi_{4 M_{3}}^{M_{2}}$ and use Corollary 1.3 to give the bound

$$
\mathscr{U}_{3} \ll\left\{X K_{3}^{-1 / 4}+X^{7 / 8}\left(M_{2} M_{3}^{-1}\right)^{3 / 8}\right\} X^{\varepsilon} .
$$

The first two terms must now be treated using results from 22] we do not use (1.8) directly, since it is slightly more efficient to use the bound

$$
\begin{aligned}
\mathfrak{S}_{2} & :=\sum_{k, l, m} g(k l m) \phi_{1}(k) \phi_{2}(l) \phi_{3}(m) a(r k l m-1) \\
& \ll\left\{\left(\frac{X}{Y}\right)^{3} r^{7 / 8} X^{7 / 8}+\left(\frac{X}{Y}\right)^{5 / 2} r^{3 / 4} X^{15 / 16}+\left(\frac{X}{Y}\right)^{2} r^{5 / 8} X^{23 / 24}\right\} X^{\varepsilon}
\end{aligned}
$$

from page 404 of [22], where $g$ is as above, and the smoothing functions separate $k, l, m$ into smoothed dyadic intervals $k \sim K, l \sim L$ and $m \sim M$ for $K L M=X$.

\footnotetext{
${ }^{2}$ The error mentioned in the footnote in the introduction affects this bound, which is used in 22 to prove Theorem 2. The bound stated here in (9.12) has been corrected.
} 
For each quadruple $k_{1}, k_{2}, k_{3}, m_{3}$ in $\mathscr{T}_{3}$ we write $r=k_{1} k_{2} k_{3} m_{3}$ and the sum over $n_{1}, n_{2}$ and $n_{3}$ is

$$
\begin{aligned}
\sum_{n_{1}, n_{2}, n_{3}} g\left(r n_{1} n_{2} n_{3}\right) \phi_{4 M_{2}}^{M_{1}}\left(k_{2} k_{3} m_{3} n_{2} n_{3}\right) \phi_{4 M_{3}}^{M_{2}}\left(k_{3} m_{3} n_{3}\right) a\left(r n_{1} n_{2} n_{3}-1\right) \\
\quad \ll\left\{\left(\frac{X}{Y}\right)^{3} X^{7 / 8}+\left(\frac{X}{Y}\right)^{5 / 2} r^{-3 / 16} X^{15 / 16}+\left(\frac{X}{Y}\right)^{2} r^{-1 / 3} X^{23 / 24}\right\} X^{\varepsilon}
\end{aligned}
$$

It follows that

$$
\mathscr{T}_{3} \ll\left\{\left(\frac{X}{Y}\right)^{3} X^{7 / 8} R+\left(\frac{X}{Y}\right)^{5 / 2} X^{15 / 16} R^{13 / 16}+\left(\frac{X}{Y}\right)^{2} X^{23 / 24} R^{2 / 3}\right\} X^{\varepsilon}
$$

where $R=K_{1} K_{2} K_{3} M_{3}$. The same arguments apply to $\mathscr{S}_{3}$, except that we use $K_{1} K_{2} K_{3}$ in place of $R$ and obtain a smaller upper bound. The first term is larger than the third only if $R>X^{-11 / 4} Y^{3}$, in which case the third term is worse than trivial. Similarly, if the second is larger than the third, then the third is worse than trivial. Thus we can ignore the first and second terms.

We now collect bounds. As already noted the error term in (9.6) is smaller than (9.10), and (9.4), (9.7) and (9.10) are all smaller than (9.13), since we have restricted the parameters so that $K_{1} K_{2} M_{3} \leq X^{1 / 3}$. We must then take into account (9.3), (9.5), (9.9), (9.11) and (9.13), and

$$
\begin{aligned}
\mathscr{T}_{f}^{*}(X) \ll\{ & K_{1}^{-1 / 4}+K_{2}^{-1 / 4}+K_{3}^{-1 / 4}+X^{1 / 4} M_{1}^{-3 / 8}+X^{-1 / 8}\left(M_{1} M_{2}^{-1}\right)^{3 / 8} \\
& \left.\quad+X^{-1 / 8}\left(M_{2} M_{3}^{-1}\right)^{3 / 8}+X^{47 / 24} Y^{-2}\left(K_{1} K_{2} K_{3} M_{3}\right)^{2 / 3}\right\} X^{1+\varepsilon}+Y X^{\varepsilon} .
\end{aligned}
$$

We now balance the terms containing the same parameters to choose their values optimally, finding, in turn,

$$
M_{1}=X^{1 / 2} M_{2}^{1 / 2}, M_{2}=X^{1 / 3} M_{3}^{2 / 3}, M_{3}=X^{-47 / 19} Y^{48 / 19}\left(K_{1} K_{2} K_{3}\right)^{-16 / 19}
$$

so

$$
\begin{aligned}
\mathscr{T}_{f}^{*}(X) \ll\left\{K_{1}^{-1 / 4}+K_{2}^{-1 / 4}\right. & +K_{3}^{-1 / 4} \\
& \left.+X^{47 / 152} Y^{-6 / 19}\left(K_{1} K_{2} K_{3}\right)^{2 / 19}\right\} X^{1+\varepsilon}+Y X^{\varepsilon} .
\end{aligned}
$$

Taking all terms involving $K_{1}, K_{2}$ or $K_{3}$ to be equal gives $K_{1}=K_{2}=K_{3}=$ $X^{-47 / 86} Y^{24 / 43}$ and hence

$$
\mathscr{T}_{f}^{*}(X) \ll\left\{X^{391 / 344} Y^{-6 / 43}+Y\right\} X^{\varepsilon} .
$$

Finally, we choose $Y=X^{391 / 392}$, so that $\mathscr{T}_{f}^{*}(X) \ll X^{391 / 392+\varepsilon}$. Note that the values of the parameters are

$$
K_{1}=K_{2}=K_{3}=X^{1 / 98}, M_{3}=X^{1 / 49}, M_{2}=X^{17 / 49}, M_{1}=X^{33 / 49},
$$

so the hypotheses on these parameters are satisfied. Theorem 1.1 for $\mathscr{T}_{f}(X)$ now follows by dyadic subdivision.

The proof of Theorem 1.1 for $\mathscr{M}_{f}(X)$ is very similar, except that (9.2) is used in place of (9.1). This produces two sums at each iteration instead of three, which differ from those for the first case only in replacing $\Lambda$ by $\mu$. Their estimation is thus the same, and since the missing sums were smaller than those appearing, with the above choices of parameters we obtain the same bound. 


\section{Proof of Lemma 2.1.}

We begin by proving (2.2); since the three cases are similar we show details for the case of $\check{f}(t)$ and omit the others. Beginning as in [6], we consider Barnes' integral

$$
\int_{0}^{\infty} K_{\nu}(x) x^{s-1} d x=2^{s-1} \Gamma\left(\frac{s+\nu}{2}\right) \Gamma\left(\frac{s-\nu}{2}\right)
$$

for $\operatorname{Re}(s \pm \nu)>0$. By Mellin inversion and changing the order of the integrations we can thus write

$$
\int_{0}^{\infty} \frac{f(x)}{x} K_{2 i t}(x) d x=\frac{1}{2 \pi i} \int_{(\sigma)} 2^{s-1} \Gamma\left(\frac{s}{2}+i t\right) \Gamma\left(\frac{s}{2}-i t\right) \int_{0}^{\infty} f(x) x^{-1-s} d x d s
$$

where $\sigma>0$. Recalling that $|t|>1$ by hypothesis we may also suppose, without loss of generality, that $t$ is positive so $t>1$. We move the line of integration left to $\operatorname{Re} s=-1$ passing two poles, one for each of the $\Gamma$-functions, and obtain

$$
\begin{array}{r}
\int_{0}^{\infty} \frac{f(x)}{x} K_{2 i t}(x) d x=2^{2 i t} \Gamma(2 i t) \int_{0}^{\infty} f(x) x^{-1-2 i t} d x+2^{-2 i t} \Gamma(-2 i t) \int_{0}^{\infty} f(x) x^{-1+2 i t} d x \\
+\frac{1}{2 \pi i} \int_{(-1)}^{\infty} 2^{s-1} \Gamma\left(\frac{s}{2}+i t\right) \Gamma\left(\frac{s}{2}-i t\right) \int_{0}^{\infty} f(x) x^{-1-s} d x d s .
\end{array}
$$

The first term can be bounded by

$$
\begin{aligned}
2^{2 i t} \Gamma(2 i t) \int_{0}^{\infty} f(x) x^{-1-2 i t} d x & \ll\{\Gamma(2 i t) \Gamma(-2 i t)\}^{1 / 2} \frac{1}{|t|} \int_{0}^{\infty}\left|f^{\prime}(x)\right| d x \\
& \ll Z \frac{\{\Gamma(2 i t) \Gamma(1-2 i t)\}^{1 / 2}}{|t||1-2 i t|^{1 / 2}}
\end{aligned}
$$

and since $|t|>1$ this is $O\left(Z|t|^{-3 / 2}(\cosh \pi t)^{-1}\right)$. The same bound holds for the second term. In the third term we integrate by parts in $s$ and use the relation $x \Gamma(x)=\Gamma(x+1)$ to obtain

$$
\begin{aligned}
-\frac{1}{2 \pi} \int_{0}^{\infty} f^{\prime}(x) x \int_{-\infty}^{\infty} & \Gamma\left(\frac{1+i \xi+2 i t}{2}\right) \Gamma\left(\frac{1+i \xi-2 i t}{2}\right) \\
& \times \frac{(x / 2)^{-i \xi} d \xi}{(1-i \xi-2 i t)(1-i \xi+2 i t)(1-i \xi)} d x
\end{aligned}
$$

and consider the inner integral. The part for $\xi>2 t$ can be estimated easily; we bound the absolute value of the integrand using the relation $|\Gamma(1 / 2+i x)|^{2}=$ $\pi / \cosh \pi x$, and obtain an upper bound

$$
\int_{2 t}^{\infty} \ll \int_{2 t}^{\infty} \frac{(\cosh \pi \xi+\cosh 2 \pi t)^{-1 / 2} d \xi}{\sqrt{\left(1+(\xi+2 t)^{2}\right)\left(1+(\xi-2 t)^{2}\right)\left(1+\xi^{2}\right)}} \ll t^{-2} \int_{2 t}^{\infty} e^{-\pi \xi / 2} d \xi \ll e^{-\pi t} t^{-2} .
$$


By symmetry the range $\xi<-2 t$ can be bounded in the same way. The integral over $(-2 t, 0)$ is the complex conjugate of that over $(0,2 t)$, so it is sufficient to consider only the latter, and splitting further at $\xi=t$ we have

$$
\int_{0}^{2 t} \ll \frac{e^{-\pi t}}{t} \int_{0}^{2 t} \frac{d \xi}{\sqrt{\left(1+(2 t-\xi)^{2}\right)\left(1+\xi^{2}\right)}} \ll \frac{e^{-\pi t}}{t^{2}} \int_{0}^{t} \frac{d \xi}{\sqrt{1+\xi^{2}}} \ll \frac{e^{-\pi t}}{t^{2}}(1+\log t) .
$$

Applying this in (10.1) the proof of $(2.2)$ for $\check{f}(t)$ is complete. As noted in the introduction, (2.3) follows from (2.2) by the same reasoning as used for (2.14) in [3]; we omit the proof.

The proof of (2.1) for $l=0$ is as for equation (7.2) in [6]. For $\hat{f}(t)$, for instance,

$$
\frac{J_{2 i t}(x)-J_{-2 i t}(x)}{\sinh \pi t}=\frac{4 i}{\pi} \int_{0}^{\infty} \cos (x \cosh \xi) \cos 2 t \xi d \xi
$$

and hence

$$
\hat{f}(t)=2 \int_{0}^{\infty} \cos 2 t \xi \int_{0}^{\infty} \frac{f(x)}{x} \cos (x \cosh \xi) d x d \xi .
$$

Splitting the integral at $\xi=A$ we treat the contribution from $0<\xi<A$ trivially, and integrate by parts in $x$ for $A<\xi<\infty$; this gives

$$
\hat{f}(t)=-2 \int_{A}^{\infty} \frac{\cos 2 t \xi}{\cosh \xi} \int_{0}^{\infty}\left(\frac{f(x)}{x}\right)^{\prime} \sin (x \cosh \xi) d x d \xi+O(A) \ll \frac{Z}{X} \int_{A}^{\infty} e^{-\xi} d \xi+A
$$

and choosing $A=\log Z / X$ if $Z>X$ and $A=0$ if $Z<X$ we obtain (2.1) for $\hat{f}(t)$ and $l=0$. A similar argument for $\check{f}(t)$ uses the integral representation

$$
K_{2 i t}(x)=\frac{1}{\cosh \pi t} \int_{0}^{\infty} \cos (x \sinh \xi) \cos 2 t \xi d \xi
$$

to give

$$
\check{f}(t)=\frac{2}{\pi} \int_{0}^{\infty} \cos 2 t \xi \int_{0}^{\infty} \frac{f(x)}{x} \cos (x \sinh \xi) d x d \xi .
$$

Separating the integral in $\xi$ at $B=\operatorname{Arsh}(Z / X)$ we obtain

$$
\check{f}(t) \ll \int_{0}^{B} d \xi+\frac{Z}{X} \int_{B}^{\infty} \frac{d \xi}{\sinh \xi}=\log \left(\frac{Z}{X}+\sqrt{1+\frac{Z^{2}}{X^{2}}}\right)+\frac{Z}{X} \log \left(\frac{X}{Z}+\sqrt{1+\frac{X^{2}}{Z^{2}}}\right)
$$

by reasoning similar to the above, and we are done. For $\tilde{f}(t)$ we use

$$
J_{t}(y)=\frac{1}{2 \pi} \int_{-\pi}^{\pi} e^{i y \sin \xi-i t \xi} d \xi-\frac{\sin \pi t}{t} \int_{0}^{\infty} e^{-y \sinh \xi-t \xi} d \xi
$$

which splits $\tilde{f}(t)$ into two parts. For the second part the argument is as for $\check{f}(t)$, splitting the integral in $\xi$ at $B$. For the first part we consider only the contribution 
from $0<\xi<\pi / 2$, since the other parts are similar, and if $Z<X$ we define $C=\arcsin (Z / X)$ and bound it as

$$
\begin{aligned}
\frac{1}{2 \pi} \int_{0}^{\pi / 2} e^{-i t \xi} \int_{0}^{\infty} \frac{f(y)}{y} e^{i y \sin \xi} d y d \xi & \ll C+\frac{Z}{X} \log \{\operatorname{cosec} C+\cot C\} \\
& \ll \frac{Z}{X}\left\{1+\log \left(\frac{X}{Z}\right)\right\}
\end{aligned}
$$

and if $Z>X$ we bound it more simply as $O(1)$. Collecting bounds we are done.

To show (2.4), note that (10.4) also gives

$$
\hat{f}(i t)=-2 \int_{0}^{\infty} \frac{f(x)}{x} \int_{0}^{\infty} \cos (x \cosh \xi) \cosh 2 t \xi d \xi d x
$$

and changing orders (which can be justified since $t \in(0,1 / 2))$ we use similar reasoning to that above to obtain

$$
\begin{aligned}
\hat{f}(i t) & \ll \int_{0}^{A} \cosh 2 t \xi d \xi+\frac{Z}{X} \int_{A}^{\infty} \frac{\cosh 2 t \xi}{\cosh \xi} d \xi \\
& \ll \begin{cases}\frac{1}{1-2 t} \frac{Z}{X} & \text { if } Z<X, \\
\left\{\frac{1}{1-2 t}+\log \frac{Z}{X}\right\}\left(\frac{Z}{X}\right)^{2 t} & \text { if } X<Z,\end{cases}
\end{aligned}
$$

where $A$ is as before. The same holds for $\check{f}(i t)$; it is easier to use the integral representation

$$
K_{-2 t}(x)=\int_{0}^{\infty} e^{-x \cosh \xi} \cosh 2 t \xi d \xi
$$

than to use (10.6) as above, but the rest of the argument is similar. The bounds (10.10) imply (2.4) for congruence groups, where $t$ is bounded away from $1 / 2$, but for the general case we must work a little harder. The Bessel functions satisfy

$$
\frac{d}{d z}\left\{z^{\nu} J_{\nu}(z)\right\}=z^{\nu} J_{\nu-1}(z), \frac{d}{d z}\left\{z^{-\nu} J_{\nu}(z)\right\}=-z^{-\nu} J_{\nu+1}(z)
$$

and using integration by parts one can show

$$
\hat{f}(i t)=\frac{\pi}{2 \sin \pi t} \int_{0}^{\infty}\left\{\frac{f^{\prime}(x)}{x}-2(1-t) \frac{f(x)}{x^{2}}\right\}\left\{J_{2 t-1}(x)+J_{1-2 t}(x)\right\} d x .
$$

If $X>Z$ the Bessel functions can be bounded as $O\left(x^{-1 / 2}\right)$, and $\hat{f}(i t) \ll|t|^{-1} Z X^{-1}$ by (10.9); using this for $|t|>\left(2+X^{1 / 2}\right)^{-1}$ and (10.10) otherwise we obtain a bound of $\hat{f}(i t) \ll Z / X$, where the implied constant is absolute. On the other hand, if $X<Z$ we use the recurrence relations

$$
J_{\nu-1}(x)-J_{\nu+1}(x)=2 J_{\nu}^{\prime}(x)
$$


to write

(10.13)

$$
\hat{f}(i t)=\frac{\pi}{2 \sin \pi t} \int_{0}^{\infty}\left\{J_{2 t}(x)-J_{2-2 t}(x)\right\} \frac{f(x)}{x} d x-\frac{\pi}{\sin \pi t} \int_{0}^{\infty} J_{1-2 t}^{\prime}(x) \frac{f(x)}{x} d x .
$$

From the series expansion

$$
J_{\nu}(x)=\sum_{l=0}^{\infty} \frac{(-1)^{l}}{l ! \Gamma(1+l+\nu)}\left(\frac{x}{2}\right)^{2 l+\nu}
$$

it follows that

$$
\left(\frac{x}{2}\right)^{\nu}\left\{\frac{1}{\Gamma(1+\nu)}-\frac{1}{\Gamma(2+\nu)}\right\} \leq J_{\nu}(x) \leq\left(\frac{x}{2}\right)^{\nu} \frac{1}{\Gamma(1+\nu)}
$$

for $x \in(0,2)$ and $\nu \in(-1,1)$, and the upper bound extends to the case where $\nu>-1 / 2$ and $x \in(0, \infty)$ by the integral representation

$$
J_{\nu}(x)=\frac{(x / 2)^{\nu}}{\Gamma(1 / 2) \Gamma(\nu+1 / 2)} \int_{-1}^{1}\left(1-\xi^{2}\right)^{\nu-1 / 2} \cos (\xi x) d \xi .
$$

The first term in (10.10) is thus $O\left(t^{-1}\right)$, and the second term is $O\left(t^{-1}(1-2 t) Z X^{-2 t}\right)$ by integration by parts. Using this bound when $1-2 t<(2+\log Z / X)^{-1}$ and (10.10) otherwise, we obtain (2.4) for $\hat{f}(i t)$. The arguments for $\check{f}(i t)$ are similar, using

$$
K_{\nu}(z)=\frac{\pi}{2 \sin \pi \nu}\left\{I_{-\nu}(z)-I_{\nu}(z)\right\}, I_{\nu}(z)=\sum_{k=0}^{\infty} \frac{(z / 2)^{\nu+2 k}}{k ! \Gamma(1+k+\nu)}
$$

and

$$
\frac{d}{d z}\left\{z^{\nu} K_{\nu}(z)\right\}=-z^{\nu} K_{\nu-1}(z), \frac{d}{d z}\left\{z^{-\nu} K_{\nu}(z)\right\}=-z^{-\nu} K_{\nu+1}(z)
$$

To show (2.1) for $l>0$ the relations (10.16) can be used together with integrations by parts to give

$$
\check{f}(t)=\frac{2}{\pi} \cosh \pi t \int_{0}^{\infty} \frac{d}{d x}\left\{\frac{d}{d x}\left\{f(x) x^{-2-2 i t}\right\} x^{4 i t+1}\right\} x^{-2 i t} K_{2 i t}(x) \frac{d x}{x}=\check{f_{1}}(t)
$$

for $f_{1}(x)=f^{\prime \prime}(x)-3 f^{\prime}(x) x^{-1}+4 f(x) x^{-2}\left(1+t^{2}\right)$. Note that $f_{1}$ has the same support as $f$, is $O\left(\left(Y^{2}+t^{2}\right) X^{-2}\right)$, and has derivatives of all orders bounded by

$$
f_{1}^{(\nu)}(x) \ll\left(\frac{Z^{2}+t^{2}}{X^{2}}\right)\left(\frac{Z}{X}\right)^{\nu}, \nu=0,1,2 \ldots
$$

We can repeat the reasoning as many times as we wish, as long as $f$ is sufficiently differentiable, to give $f_{2}, f_{3}, \ldots, f_{l}$, and applying the bound for $l=0$ to $\check{f}_{l}(t)$ proves the general case for $\check{f}(t)$. The other cases are similar.

To prove (2.5) for $\hat{f}(t)$ note first that

$$
J_{-2 t}(x)-J_{2 t}(x) \geq\left(\frac{x}{2}\right)^{-2 t}\left\{\frac{1}{2 \Gamma(1-2 t)}-\frac{1}{\Gamma(1+2 t)}\left(\frac{x}{2}\right)^{4 t_{0}}\right\}
$$


for $t \in\left(t_{0}, 1 / 4\right)$ for fixed $t_{0}>0$, from (10.14), so

$$
-\hat{f}(i t)=\frac{1}{2 \sin \pi t} \int_{0}^{\infty}\left\{J_{-2 t}(x)-J_{2 t}(x)\right\} f(x) \frac{d x}{x} \asymp t^{-1}\left(\frac{X}{2}\right)^{-2 t} \int_{0}^{\infty} f(x) \frac{d x}{x} .
$$

If $X$ is sufficiently small (depending on $t_{0}$ ), then this implies $(2.5)$ for $t \in\left(t_{0}, 1 / 4\right)$. To complete the proof for all $t \in(0,1 / 4)$ requires analysing the series more closely for small $t$. Still restricting to $t \in(0,1 / 4)$, the bounds

$$
\left|\prod_{k=1}^{l}(k \pm 2 t)-l !\right| \leq t 2^{l+1} l !, \prod_{k=1}^{l}(k+2 t) \geq l !, \prod_{k=1}^{l}(k-2 t) \geq(1-2 t)(l-1) !
$$

imply

$$
\begin{array}{r}
\frac{J_{-2 t}(x)-J_{2 t}(x)}{\sin \pi t}=\frac{1}{\sin \pi t}\left\{\frac{(x / 2)^{-2 t}}{\Gamma(1-2 t)}-\frac{(x / 2)^{2 t}}{\Gamma(1+2 t)}\right\} \sum_{l=0}^{\infty} \frac{(-1)^{l}(x / 2)^{2 l}}{\prod_{k=1}^{l}\left(k^{2}-4 t^{2}\right)} \\
+O\left(\left\{\frac{(x / 2)^{2-2 t}}{\Gamma(2-2 t)}+\frac{(x / 2)^{2+2 t}}{(1-2 t) \Gamma(1+2 t)}\right\} e^{x^{2} / 2}\right)
\end{array}
$$

and for $x<1$ the error term can be replaced by $O\left(x^{2-2 t}\right)$, where the implied constant is absolute. Since $\Gamma(1+x)=1-\gamma x+O\left(x^{2}\right)$, where $\gamma=0.57721 \ldots$ is Euler's constant, if we suppose that $x \leq 2 e^{-2 \gamma}$, then

$$
\begin{aligned}
& \frac{1}{\sin \pi t}\left\{\frac{(x / 2)^{-2 t}}{\Gamma(1-2 t)}-\frac{(x / 2)^{2 t}}{\Gamma(1+2 t)}\right\} \\
= & \left(\frac{x}{2}\right)^{-2 t} \frac{\cos \pi t}{\pi t}\left\{\Gamma(1+2 t)-\Gamma(1-2 t)(x / 2)^{4 t}\right\} \\
= & \left(\frac{x}{2}\right)^{-2 t} \frac{\cos \pi t}{\pi}\left\{\frac{1-(x / 2)^{4 t}}{t}-2 \gamma\left(1+(x / 2)^{4 t}\right)+O(t)\right\} \\
\geq & \left(\frac{x}{2}\right)^{-2 t} \frac{\cos \pi t}{\pi}\{4 \gamma+O(t)\},
\end{aligned}
$$

so there exists $t_{0}$ such that for $t \in\left(0, t_{0}\right)$ and for all $x \in\left(0,2 e^{-2 \gamma}\right)$ this is at least $(x / 2)^{-2 t} / 2 \pi$, say. Since

$$
\sum_{l=0}^{\infty} \frac{(-1)^{l}(x / 2)^{2 l}}{\prod_{k=1}^{l}(k-2 t)(k+2 t)} \geq 1-\frac{(x / 2)^{2}}{1-4 t^{2}} \geq 1-\frac{4}{3} e^{-4 \gamma}>\frac{2}{3}
$$

this gives a lower bound

$$
\frac{J_{-2 t}(x)-J_{2 t}(x)}{\sin \pi t} \geq \frac{1}{3 \pi}\left(\frac{x}{2}\right)^{-2 t}+O\left(x^{2-2 t}\right) \geq \frac{1}{6 \pi}\left(\frac{x}{2}\right)^{-2 t}
$$

for $t \in\left(0, t_{0}\right)$ and $x$ sufficiently small. The proof of $(2.5)$ is now completed by using this estimate in the definition of $\hat{f}(t)$. The argument for $\check{f}(t)$ is similar, using (10.15). This completes the proof of Lemma 2.1 .

\section{ACKNOWLEDGMENTS}

The author thanks Henryk Iwaniec for suggesting this problem many years ago and for his support during and since the elaboration of [21, where some of the ideas related to Theorem 1.4 originated. Most of this work was completed and 
the paper written in the spring of 2011, while the author was a member at the Institute for Advanced Study in Princeton. He thanks the Institute for the support received and for the opportunity to spend time in such a stimulating environment. Particular thanks are due to Enrico Bombieri and Peter Sarnak for their time and encouragement. The author also thanks the referees, whose detailed comments were extremely helpful in correcting and improving this paper.

\section{REFERENCES}

[1] Valentin Blomer, Shifted convolution sums and subconvexity bounds for automorphic Lfunctions, Int. Math. Res. Not. 73 (2004), 3905-3926, DOI 10.1155/S1073792804142505. MR2104288(2005m:11090)

[2] Valentin Blomer and Gergely Harcos, The spectral decomposition of shifted convolution sums, Duke Math. J. 144 (2008), no. 2, 321-339, DOI 10.1215/00127094-2008-038. MR 2437682 (2009k:11087)

[3] V. Blomer, G. Harcos, and P. Michel, A Burgess-like subconvex bound for twisted L-functions, Forum Math. 19 (2007), no. 1, 61-105, DOI 10.1515/FORUM.2007.003. Appendix 2 by Z. Mao. MR2296066 (2008i:11067)

[4] E. Bombieri, J. B. Friedlander, and H. Iwaniec, Primes in arithmetic progressions to large moduli, Acta Math. 156 (1986), no. 3-4, 203-251, DOI 10.1007/BF02399204. MR834613 (88b:11058)

[5] Pierre Deligne, La conjecture de Weil. I, Inst. Hautes Études Sci. Publ. Math. 43 (1974), 273-307 (French). MR0340258(49 \#5013)

[6] J.-M. Deshouillers and H. Iwaniec, Kloosterman sums and Fourier coefficients of cusp forms, Invent. Math. 70 (1982/83), no. 2, 219-288, DOI 10.1007/BF01390728. MR684172 (84m:10015)

[7] W. Duke, J. B. Friedlander, and H. Iwaniec, A quadratic divisor problem, Invent. Math. 115 (1994), no. 2, 209-217, DOI 10.1007/BF01231758. MR1258903 (95d:11122)

[8] Étienne Fouvry, Sur le problème des diviseurs de Titchmarsh, J. Reine Angew. Math. 357 (1985), 51-76, DOI 10.1515/crll.1985.357.51 (French). MR783533 (87b:11090)

[9] Anton Good, On various means involving the Fourier coefficients of cusp forms, Math. Z. 183 (1983), no. 1, 95-129, DOI 10.1007/BF01187218. MR701361 (84g:10052)

[10] James Lee Hafner, Explicit estimates in the arithmetic theory of cusp forms and Poincaré series, Math. Ann. 264 (1983), no. 1, 9-20, DOI 10.1007/BF01458047. MR709858(85f:11032a)

[11] H. Halberstam, Footnote to the Titchmarsh-Linnik divisor problem, Proc. Amer. Math. Soc. 18 (1967), 187-188. MR0204379 (34 \#4221)

[12] Gergely Harcos, An additive problem in the Fourier coefficients of cusp forms, Math. Ann. 326 (2003), no. 2, 347-365, DOI 10.1007/s00208-003-0421-1. MR1990914(2004d:11029)

[13] Henryk Iwaniec and Emmanuel Kowalski, Analytic number theory, American Mathematical Society Colloquium Publications, vol. 53, American Mathematical Society, Providence, RI, 2004. MR2061214 (2005h:11005)

[14] M. Jutila, Transformations of exponential sums, Proceedings of the Amalfi Conference on Analytic Number Theory (Maiori, 1989), Univ. Salerno, Salerno, 1992, pp. 263-270. MR1220470 (94i:11059)

[15] M. Jutila, A variant of the circle method, Sieve methods, exponential sums, and their applications in number theory (Cardiff, 1995), London Math. Soc. Lecture Note Ser., vol. 237, Cambridge Univ. Press, Cambridge, 1997, pp. 245-254, DOI 10.1017/CBO9780511526091.016. MR:1635766 (99f:11134)

[16] Matti Jutila, The additive divisor problem and its analogs for Fourier coefficients of cusp forms. I, Math. Z. 223 (1996), no. 3, 435-461, DOI 10.1007/PL00004270. MR.1417854 (98b:11046)

[17] Matti Jutila, The additive divisor problem and its analogs for Fourier coefficients of cusp forms. II, Math. Z. 225 (1997), no. 4, 625-637, DOI 10.1007/PL00004323. MR.1466405 (98i:11026)

[18] H. H. Kim, Functoriality for the exterior square of GL4 and the symmetric fourth of GL2, with Appendix 1 by D. Ramakrishnan and Appendix 2 by H. Kim and P. Sarnak, 
J. Amer. Math. Soc. 16 (2003), 139-183, DOI 10.1090/S0894-0347-02-00410-1. MR.1937203 (2003k:11083)

[19] N. V. Kuznecov, The Petersson conjecture for cusp forms of weight zero and the Linnik conjecture. Sums of Kloosterman sums, Mat. Sb. (N.S.) 111(153) (1980), no. 3, 334-383, 479 (Russian). MR.568983 (81m:10053)

[20] Ju. V. Linnik, The dispersion method in binary additive problems, Translated by S. Schuur, American Mathematical Society, Providence, R.I., 1963. MR0168543 (29 \#5804)

[21] Nigel John E. Pitt, Convolutions of automorphic L-series, ProQuest LLC, Ann Arbor, MI, 1992. Thesis (Ph.D.)-Rutgers The State University of New Jersey - New Brunswick. MR2716401

[22] Nigel J. E. Pitt, On shifted convolutions of $\zeta^{3}(s)$ with automorphic L-functions, Duke Math. J. 77 (1995), no. 2, 383-406, DOI 10.1215/S0012-7094-95-07711-4. MR1321063 (97a:11069)

[23] Gaetano Rodriquez, Sul problema dei divisori di Titchmarsh, Boll. Un. Mat. Ital. (3) 20 (1965), 358-366 (Italian, with English summary). MR0197409 (33 \#5574)

[24] P. Sarnak, Three lectures on the Möbius function Randomness and Dynamics, available at http://www.math.ias.edu/wam/2011/lectures.

[25] Peter Sarnak, Estimates for Rankin-Selberg L-functions and quantum unique ergodicity, J. Funct. Anal. 184 (2001), no. 2, 419-453, DOI 10.1006/jfan.2001.3783. MR.1851004 (2003c:11050)

[26] Peter Sarnak, Integrals of products of eigenfunctions, Internat. Math. Res. Notices 6 (1994), $251 \mathrm{ff}$., approx. 10 pp. (electronic), DOI 10.1155/S1073792894000280. MR1277052(95i:11039)

[27] Atle Selberg, On the estimation of Fourier coefficients of modular forms, Proc. Sympos. Pure Math., Vol. VIII, Amer. Math. Soc., Providence, R.I., 1965, pp. 1-15. MR0182610 (32 \#93)

[28] E. C. Titchmarsh, A divisor problem, Rend. Circ. Mat. Palermo 54 (1930), 414-429.

[29] E. C. Titchmarsh, A divisor problem. Correction, Rend. Circ. Mat. Palermo 57 (1933), 478479.

[30] Robert-C. Vaughan, Sommes trigonométriques sur les nombres premiers, C. R. Acad. Sci. Paris Sér. A-B 285 (1977), no. 16, A981-A983 (French, with English summary). MR0498434 (58 \#16555)

Departamento de Matemática, Universidade de Brasília, DF 70910-900, Brazil

E-mail address: pitt@mat.unb.br 